QUARTERLY OF APPLIED MATHEMATICS

VOLUME LXVIII, NUMBER 3

SEPTEMBER 2010, PAGES 513-536

S 0033-569X(2010)01171-5

Article electronically published on May 27, 2010

\title{
LOW-FREQUENCY DIPOLAR EXCITATION OF A PERFECT ELLIPSOIDAL CONDUCTOR
}

\author{
$\mathrm{BY}$ \\ GAËLE PERRUSSON (Département de Recherche en Electromagnétisme - Laboratoire des Signaux \\ et Systèmes, (Univ. Paris-Sud, CNRS, SUPELEC) UMR8506, 3 rue Joliot-Curie, Gif-sur-Yvette, \\ F-91192, France), \\ PANAYIOTIS VAFEAS (Division of Applied Mathematics and Mechanics - Department of \\ Engineering Sciences, School of Engineering - University of Patras, Patras 265 04, Greece), \\ AND \\ DOMINIQUE LESSELIER (Département de Recherche en Electromagnétisme - Laboratoire des \\ Signaux et Systèmes, (CNRS, Univ. Paris-Sud, SUPELEC) UMR8506, 3 rue Joliot-Curie, \\ Gif-sur-Yvette, F-91192, France)
}

Abstract. This paper deals with the scattering by a perfectly conductive ellipsoid under magnetic dipolar excitation at low frequency. The source and the ellipsoid are embedded in an infinite homogeneous conducting ground. The main idea is to obtain an analytical solution of this scattering problem in order to have a fast numerical estimation of the scattered field that can be useful for real data inversion. Maxwell equations and boundary conditions, describing the problem, are firstly expanded using low-frequency expansion of the fields up to order three. It will be shown that fields have to be found incrementally. The static one (term of order zero) satisfies the Laplace equation. The next non-zero term (term of order two) is more complicated and satisfies the Poisson equation. The order-three term is independent of the previous ones and is described by the Laplace equation. They constitute three different scattering problems that are solved using the separated variables method in the ellipsoidal coordinate system. Solutions are written as expansions on the few analytically known scalar ellipsoidal harmonics. Details are given to explain how those solutions are achieved with an example of numerical results.

1. Introduction. This paper describes how to obtain an approximate solution of the field scattered by an impenetrable ellipsoid illuminated by a magnetic dipole at low

Received November 20, 2008.

2000 Mathematics Subject Classification. Primary 78A45; Secondary 78A25.

Key words and phrases. Low-frequency expansion, ellipsoidal harmonics, dipole excitation.

E-mail address: perrusson@lss.supelec.fr

E-mail address: vafeas@des.upatras.gr

E-mail address: lesselier@lss.supelec.fr

(C) 2010 Brown University Reverts to public domain 28 years from publication 
frequencies. This work is motivated by geophysical applications [1, 2] (mining prospection), 3] (detection of cavity), or other underground detections 4, [5] (UXO - Unexploded Ordnance) [6], where one wants to localize and/or to estimate size, orientation and conductivity of the embedded structure. Generally, an inverse scheme is used to find either a smooth surface of the structure 7 or the parameters of an equivalent object [8], 9] using iterative methods. Most of the proposed solutions use meshing methods for the scattering problem that have to be evaluated at each iteration, resulting in a large time-consuming numerical process. In practice, users want to measure and to identify the structure in-situ at the same time. For this reason, inverse schemes have to be fast, which can be achieved only if the direct problem is itself very fast. In order to reach this objective, analytical solutions of the scattering problem can be of good help. C. Ao and colleagues provide in [10] the static solution of the quasi-magnetostatic problem for the scattering by a spheroid. The case of an ellipsoid with a low conductivity compared to the ground is given in [11] where the solution is based on the localized non-linear approximation [12. In this paper we will discuss the impenetrable ellipsoidal case in order to deal with the very high contrast of conductivity of the structure compared to the underground.

The ellipsoidal geometry has been chosen because, for such applications, the source works at low frequencies in order to deeply penetrate in the conductive ground. Actually, at such frequencies, measurements are only sensitive to general information about the structure like global shape, average conductivity, etc. Therefore, the equivalent object must have a general shape in order to yield information such as orientation and size, but should not be too complicated, either. The ellipsoid has the advantage of being one of the most general canonical shapes. Generally the source is a magnetic dipole located in a borehole or a loop on the ground that can be approximated by a magnetic dipole as a first approximation. For all these reasons, the authors decided to provide an efficient solution of the scattering by a perfectly conducting ellipsoid illuminated by a magnetic dipole. In this paper, the source and the ellipsoid are supposed to be located in an infinite homogeneous conductive ground. This simplified environment constitutes a reasonable approximation if both the source and the dipole are far enough from the surface of the ground. Therefore, if measurements are done in a borehole, then this approximation is reasonably valid.

In Part 2 the scattering problem is first described and simplified as a low-frequency problem using low-frequency expansions. The authors will explain why such approximations are needed to solve the problem in the ellipsoidal coordinate system using the few scalar ellipsoidal harmonics known. The third part explains how the solution is obtained for each term of the low-frequency expansion. Actually, some of them satisfy a Laplace equation and others the Poisson one, with very different incident fields so that calculations must be explained separately. Choices that have been made to correctly describe the order-two fields and that are far from trivial, due to the lack of information about ellipsoidal harmonics, are also explained. Numerical results are given in Part 4 in order to emphasize how such a contribution can be useful. Part 5 provides the authors' conclusions. 


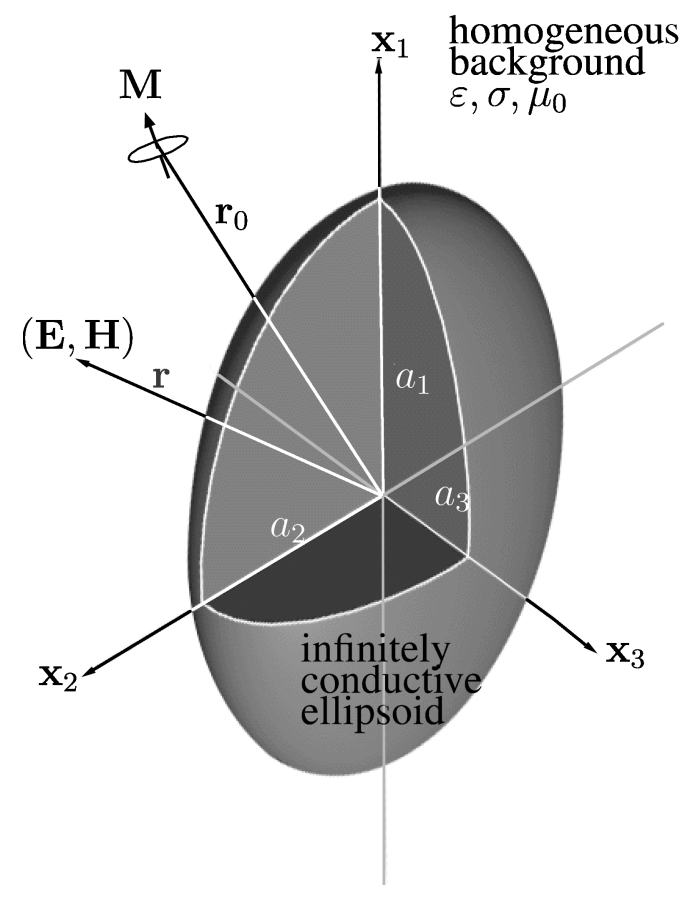

FIG. 1. Problem geometry

2. Low-frequency scattering problem. The problem considered here is one of a perfectly conducting ellipsoid illuminated by a magnetic dipole $\mathbf{M}$ with time-harmonic dependence $\exp (-\mathrm{j} \omega t)$, where $\omega$ is the angular frequency. The ellipsoid is located at the center of a Cartesian coordinate system $\left(\mathbf{x}_{1}, \mathbf{x}_{2}, \mathbf{x}_{3}\right)$, each axis being along one of the semi-axes $a_{1}>a_{2}>a_{3}$ of the ellipsoid, as shown in Figure 1. The magnetic dipole is located at $\mathbf{r}_{0}$ and is defined by its vector intensity $\mathbf{M}=M_{1} \mathbf{x}_{1}+M_{2} \mathbf{x}_{2}+M_{3} \mathbf{x}_{3}$ that can be of any orientation.

The wavenumber $k$ around the ellipsoid is defined by $k^{2}=\varepsilon \mu_{0} \omega^{2}+\mathrm{j} \omega \mu_{0} \sigma$, where $\varepsilon$ and $\sigma$ are the dielectric permittivity and the static conductivity of the surrounding medium, and $\mu_{0}$ is the permeability of vacuum.

The incident field radiated by the magnetic dipole is well-known:

$$
\begin{aligned}
& \mathbf{E}^{i}(\mathbf{r})=-\mathrm{j} \omega \mu_{0} \frac{\mathbf{M}}{4 \pi} \times\left[-\frac{\mathbf{R}}{R^{3}}+(\mathrm{j} k) \frac{\mathbf{R}}{R^{2}}\right] e^{\mathrm{j} k R} \\
& \mathbf{H}^{i}(\mathbf{r})=\left[\left(k^{2}+\frac{\mathrm{j} k}{R}-\frac{1}{R^{2}}\right) \mathbf{M}-\left(k^{2}+\frac{3 \mathrm{j} k}{R}-\frac{3}{R^{2}}\right) \frac{\mathbf{R R} \cdot \mathbf{M}}{R^{2}}\right] \frac{e^{\mathrm{j} k R}}{4 \pi R}
\end{aligned}
$$

with $\mathbf{R}=\mathbf{r}-\mathbf{r}_{0}$,

while the scattering problem is described by Maxwell's equations

$$
\begin{array}{ll}
\nabla \times \mathbf{E}^{*}(\mathbf{r})=\mathrm{j} \omega \mu_{0} \mathbf{H}^{*}(\mathbf{r}) & \nabla \cdot \mathbf{E}^{*}(\mathbf{r})=0 \\
\nabla \times \mathbf{H}^{*}(\mathbf{r})=-\mathrm{j} \frac{k^{2}}{\omega \mu_{0}} \mathbf{E}^{*}(\mathbf{r}) & \nabla \cdot \mathbf{H}^{*}(\mathbf{r})=0
\end{array}
$$


(with $*=i$, or $t$ ), proper condition of radiation at infinity, and boundary conditions at the surface $S$ of the ellipsoid

$$
\mathbf{n} \times\left.\mathbf{E}^{t}(\mathbf{r})\right|_{S}=\left.\mathbf{0} \quad \mathbf{n} \cdot \mathbf{H}^{t}(\mathbf{r})\right|_{S}=0,
$$

where $\left(\mathbf{E}^{t}, \mathbf{H}^{t}\right)$ is the total field, $\left(\mathbf{E}^{i}, \mathbf{H}^{i}\right)$ the incident field, and $\mathbf{n}$ the outward-pointing unit vector, normal to the surface $S$. Boundary equations come from the physical properties of the ellipsoid, which is a perfect conductor.

In a scattering problem, the total field $\left(\mathbf{E}^{t}, \mathbf{H}^{t}\right)$ can be viewed as the superposition of the incident field $\left(\mathbf{E}^{i}, \mathbf{H}^{i}\right)$ and the scattered field $\left(\mathbf{E}^{s}, \mathbf{H}^{s}\right)$. In this paper, the authors focus their work on finding the scattered field, knowing that the total field can be easily obtained. Note that this scattered field $\left(\mathbf{E}^{s}, \mathbf{H}^{s}\right)$ also satisfies the Maxwell equations and the radiation condition at infinity, while the boundary conditions are only satisfied by the total field.

One usually solves these equations using separated solutions in the appropriate separable coordinate system. Boundary conditions are to be satisfied on the ellipsoidal surface, so the matched coordinate system is the ellipsoidal one, which is also a separable one. Then, the solution for the vector waves has to be written in terms of vector harmonics. Due to the fact that the vector ellipsoidal harmonics are unknown, no solution can be obtained analytically in terms of vector waves. Having an analytical expression of the fields in order to have a fast numerical evaluation is the main goal of this paper. If the problem cannot be solved analytically with vector ellipsoidal harmonics, scalar ones can be used if they exist. Knowing that some of those scalar ellipsoidal harmonics have been established in a simple analytical form [13, [14, 15], the authors decided to simplify the scattering problem in order to find an approximate solution using these scalar harmonics.

In a previous paper [16, for the sphere case, the authors have shown that low-frequency (LF) expansions can be used and applied to fields and quantities involved. All of the scalar or vectors are written as summations in terms of powers of $(\mathrm{j} k)$, where $k$ is the wave number in the surrounding medium and $\mathrm{j}$ is the complex number satisfying $\mathrm{j}^{2}=-1$. So for any given vector field $\boldsymbol{\psi}$ the LF expansion is $\boldsymbol{\psi}(\mathbf{r})=\sum_{l=0}^{\infty} \boldsymbol{\psi}_{l}(\mathbf{r})(\mathrm{j} k)^{l}$. It is assumed that for the low-frequency applications involved, only the first four terms contribute to the behavior of the fields. For this reason all LF expansions are truncated at $l=3$.

After working on the previous equations (11 2) and dealing with this LF expansion, plus the following one dealing with pulsation:

$$
\mathrm{j} \omega=\frac{-1}{\sigma \mu_{0}}(\mathrm{j} k)^{2}+\frac{\varepsilon}{\sigma^{3} \mu_{0}^{2}}(\mathrm{j} k)^{4},
$$

Maxwell's equations become a system of equations 1

$$
\begin{array}{llll}
\mathbf{E}_{0}^{*}(\mathbf{r})=\mathbf{0} & \nabla \cdot \mathbf{E}_{0}^{*}(\mathbf{r})=0 & \nabla \times \mathbf{H}_{0}^{*}(\mathbf{r})=\mathbf{0} & \nabla \cdot \mathbf{H}_{0}^{*}(\mathbf{r})=0 \\
\mathbf{E}_{1}^{*}(\mathbf{r})=\mathbf{0} & \nabla \cdot \mathbf{E}_{1}^{*}(\mathbf{r})=0 & \mathbf{H}_{1}^{*}(\mathbf{r})=\mathbf{0} & \nabla \cdot \mathbf{H}_{1}^{*}(\mathbf{r})=0 \\
\nabla \times \mathbf{E}_{2}^{*}(\mathbf{r})=-\frac{\mathbf{H}_{0}^{*}(\mathbf{r})}{\sigma} & \nabla \cdot \mathbf{E}_{2}^{*}(\mathbf{r})=0 & \nabla \times \mathbf{H}_{2}^{*}(\mathbf{r})=\sigma \mathbf{E}_{2}^{*}(\mathbf{r}) & \nabla \cdot \mathbf{H}_{2}^{*}(\mathbf{r})=0 \\
\mathbf{E}_{3}^{*}(\mathbf{r})=\mathbf{0} & \nabla \cdot \mathbf{E}_{3}^{*}(\mathbf{r})=0 & \nabla \times \mathbf{H}_{3}^{*}(\mathbf{r})=\mathbf{0} & \nabla \cdot \mathbf{H}_{3}^{*}(\mathbf{r})=0
\end{array}
$$

${ }^{1}$ In fact, $\varepsilon$ does not appear until the order-four field and thus does not play any role at low frequencies as is hoped for. 
that has to be satisfied by the total, incident, and scattered fields $(*=i, t, s)$, while the expansion of the incident field is

$$
\begin{array}{lrl}
\mathbf{E}_{0}^{i}(\mathbf{r})=\mathbf{0} & \mathbf{H}_{0}^{i}(\mathbf{r})=\frac{\mathbf{M}}{4 \pi} \cdot\left(\frac{3 \mathbf{R R}}{R^{2}}-\overline{\overline{\mathrm{I}}}\right) \frac{1}{R^{3}} \\
\mathbf{E}_{1}^{i}(\mathbf{r})=\mathbf{0} & \mathbf{H}_{1}^{i}(\mathbf{r})=\mathbf{0} \\
\mathbf{E}_{2}^{i}(\mathbf{r})=-\frac{1}{\sigma} \frac{\mathbf{M}}{4 \pi} \times \frac{\mathbf{R}}{R^{3}} & \mathbf{H}_{2}^{i}(\mathbf{r})=-\frac{\mathbf{M}}{4 \pi} \cdot\left(\frac{\mathbf{R R}}{R^{2}}+\overline{\overline{\mathrm{I}}}\right) \frac{1}{2 R} \\
\mathbf{E}_{3}^{i}(\mathbf{r})=\mathbf{0} & \mathbf{H}_{3}^{i}(\mathbf{r})=-\frac{2}{3} \frac{\mathbf{M}}{4 \pi}
\end{array}
$$

where $\overline{\overline{\mathrm{I}}}$ is the unit dyad.

Boundary conditions must be satisfied, which provides for the LF terms of the total field $\left(\mathbf{E}_{l}^{t}, \mathbf{H}_{l}^{t}\right)$ the following new boundary conditions

$$
\mathbf{n} \times\left.\mathbf{E}_{l}^{t}(\mathbf{r})\right|_{S}=\mathbf{0},\left.\quad \mathbf{n} \cdot \mathbf{H}_{l}^{t}(\mathbf{r})\right|_{S}=0 \quad(\text { for } l=0 \text { to } 3) .
$$

Looking at the equations in (5), one can notice that solutions can been found incrementally. The static term $\left(\mathbf{H}_{0}^{s}\right)$ has already been found by the authors [11, and details are found in Appendix $\mathrm{B}$ for completeness. Then the second-order term $\left(\mathbf{H}_{2}^{s}, \mathbf{E}_{2}^{s}\right)$ has to be found using the static solution. The third-order term is not linked to the previous one and has to satisfy simple equations under a uniform incident field $\left(\mathbf{H}_{3}^{i}\right.$ is independent of $\mathbf{r})$. At this point, the expansion of the solution, thinking that for low-frequency applications these three terms are indeed sufficient, ends as illustrated numerically thereafter.

\section{Approximate solution.}

3.1. The ellipsoidal geometry. To start with, some explanations are needed about the ellipsoidal coordinate system.

An ellipsoid is defined by its semi-axis $a_{1}>a_{2}>a_{3}$ and also its foci $h_{1}, h_{2}, h_{3}$, where

$$
h_{i}^{2}=(-1)^{i-1}\left(a_{i+1}^{2}-a_{i+2}^{2}\right) .
$$

In an ellipsoidal coordinate system, the appropriate coordinates are $(\rho, \mu, \nu)$ such that

$$
\infty>\rho^{2} \geq h_{2}^{2} \geq \mu^{2} \geq h_{3}^{2} \geq \nu^{2} \geq 0 .
$$

They satisfy the equations of the ellipsoidal coordinate surfaces (ellipsoids- $\rho$, hyperboloids of one sheet $-\mu$, hyperboloids of two sheets $-\nu$ )

$$
\sum_{i=1}^{3} \frac{x_{i}^{2}}{\theta^{2}-a_{1}^{2}+a_{i}^{2}}=1 \quad(\text { for } \theta=\rho, \mu, \nu),
$$

where the $\left(x_{1}, x_{2}, x_{3}\right)$ are the Cartesian coordinates as defined previously. The unit ellipsoidal coordinate vectors $(\boldsymbol{\rho}, \boldsymbol{\mu}, \boldsymbol{\nu})$ corresponding to ellipsoidal coordinates $(\rho, \mu, \nu)$ are orthogonal to the $\rho, \mu, \nu$ surfaces. They are linked to the Cartesian unit vectors as follows:

$$
\boldsymbol{\theta}=\frac{\theta}{h_{\theta}} \sum_{i=1}^{3} \frac{x_{i}}{\theta^{2}-a_{1}^{2}+a_{i}^{2}} \mathbf{x}_{i}
$$


where $(\boldsymbol{\theta}, \theta)$ is respectively $(\boldsymbol{\rho}, \rho),(\boldsymbol{\mu}, \mu)$, and $(\boldsymbol{\nu}, \nu)$, while $h_{\rho}, h_{\mu}, h_{\nu}$ are metric parameters such that

$$
h_{\rho}^{2}=\frac{\left(\rho^{2}-\mu^{2}\right)\left(\rho^{2}-\nu^{2}\right)}{\left(\rho^{2}-h_{3}^{2}\right)\left(\rho^{2}-h_{2}^{2}\right)}, \quad h_{\mu}^{2}=\frac{\left(\rho^{2}-\mu^{2}\right)\left(\mu^{2}-\nu^{2}\right)}{\left(\mu^{2}-h_{3}^{2}\right)\left(h_{2}^{2}-\mu^{2}\right)}, \quad h_{\nu}^{2}=\frac{\left(\rho^{2}-\nu^{2}\right)\left(\mu^{2}-\nu^{2}\right)}{\left(h_{3}^{2}-\nu^{2}\right)\left(h_{2}^{2}-\nu^{2}\right)} .
$$

It is also useful to know the expression of the Cartesian coordinates in terms of the ellipsoidal ones:

$$
x_{i}=\frac{h_{i}}{h_{1} h_{2} h_{3}}\left[(-1)^{i-1}\left(\rho^{2}-a_{1}^{2}+a_{i}^{2}\right)\left(\mu^{2}-a_{1}^{2}+a_{i}^{2}\right)\left(\nu^{2}-a_{1}^{2}+a_{i}^{2}\right)\right]^{1 / 2} .
$$

In this particular coordinate system, the gradient operator is defined by

$$
\nabla_{\mathbf{r}}=\frac{\boldsymbol{\rho}}{h_{\rho}} \frac{\partial}{\partial \rho}+\frac{\boldsymbol{\mu}}{h_{\mu}} \frac{\partial}{\partial \mu}+\frac{\boldsymbol{\nu}}{h_{\nu}} \frac{\partial}{\partial \nu} .
$$

For more details about the ellipsoidal coordinate system and operators, please refer to [17.

The expansion of the inverse distance on scalar external $\mathbb{F}_{n}^{m}(\mathbf{r})$ and internal $\mathbb{E}_{n}^{m}(\mathbf{r})$ ellipsoidal harmonics is known, and leads to

$$
\frac{1}{\left|\mathbf{r}-\mathbf{r}_{0}\right|}=\sum_{n=0}^{\infty} \sum_{m=1}^{2 n+1} \frac{1}{(2 n+1) \gamma_{n}^{m}} \mathbb{F}_{n}^{m}\left(\mathbf{r}_{0}\right) \mathbb{E}_{n}^{m}(\mathbf{r}) \quad \text { for } \rho<\rho_{0}
$$

where the $\gamma_{n}^{m}$ are constants listed in Appendix $\mathrm{A}$ with the ellipsoidal harmonics.

3.2. Solution of the third-order term. How to get the third-order term is firstly described due to its simplicity.

As said previously, fields of order three do not depend upon the fields of other orders. This is summarized by the equations of order three extracted from equations (5) and (6):

$$
\begin{array}{lrlr}
\mathbf{H}_{3}^{i}(\mathbf{r})=-\frac{2}{3} \frac{\mathbf{M}}{4 \pi} & \nabla \times \mathbf{H}_{3}^{*}(\mathbf{r})=\sigma \mathbf{E}_{3}^{*}(\mathbf{r}) & \nabla \cdot \mathbf{H}_{3}^{*}(\mathbf{r})=0 & (*=t, i, s) \\
\mathbf{E}_{3}^{i}(\mathbf{r})=\mathbf{0} & \mathbf{E}_{3}^{t}(\mathbf{r})=\mathbf{0} & \mathbf{E}_{3}^{s}(\mathbf{r})=\mathbf{0}
\end{array}
$$

The cancellation of the electric field implies that the magnetic field has zero curl everywhere. Its divergence is also null; therefore, it can be represented as the gradient of a scalar potential $\phi_{3}$ that satisfies the following equations:

$$
\mathbf{H}_{3}^{s}(\mathbf{r})=\nabla \phi_{3}(\mathbf{r}), \quad \nabla \times \mathbf{H}_{3}^{s}(\mathbf{r})=\nabla \times \nabla \phi_{3}(\mathbf{r})=\Delta \phi_{3}(\mathbf{r})=\mathbf{0}, \quad \nabla \cdot \mathbf{H}_{3}^{s}(\mathbf{r})=0 .
$$

The source is a magnetic dipole $\mathbf{M}$ of any orientation so that it can be written as a superposition of three dipoles oriented along each Cartesian vector $\mathbf{M}=\sum_{q=1}^{3} M_{q} \mathbf{x}_{q}$. Using the property of superposition, the scattered field $\mathbf{H}_{3}^{s}(\mathbf{r})$ can be expressed as the summation of gradients of three different potentials corresponding to each illumination:

$$
\mathbf{H}_{3}^{s}(\mathbf{r})=\sum_{q=1}^{3} \frac{M_{q}}{4 \pi} \nabla \phi_{3 q}(\mathbf{r}) .
$$

For convenience the constant $4 \pi$ is extracted from the potential.

The incident field can be rewritten as follows:

$$
\mathbf{H}_{3}^{i}(\mathbf{r})=-\frac{2}{3} \frac{\mathbf{M}}{4 \pi}=-\frac{2}{3} \sum_{q=1}^{3} \frac{M_{q}}{4 \pi} \mathbf{x}_{q} \mathbb{E}_{0}^{1}(\mathbf{r})=-\frac{2}{3} \sum_{q=1}^{3} \frac{M_{q}}{4 \pi} \mathbf{x}_{q},
$$


knowing that the harmonic $\mathbb{E}_{0}^{1}(\mathbf{r})$ is the unit constant.

Outside the ellipsoid, the scalar potential $\phi_{3 q}$ can be written in terms of the exterior ellipsoidal harmonics $\mathbb{F}_{n}^{m}(\mathbf{r})$ or directly in terms of the internal ellipsoidal harmonics $\mathbb{E}_{n}^{m}(\mathbf{r})$ and elliptical integrals $I_{n}^{m}(\rho)$ using equation (50):

$$
\phi_{3 q}(\mathbf{r})=\sum_{n=0}^{3} \sum_{m=1}^{2 n+1} g_{n}^{m, q} \mathbb{F}_{n}^{m}(\mathbf{r})=\sum_{n=0}^{3} \sum_{m=1}^{2 n+1}(2 n+1) g_{n}^{m, q} \mathbb{E}_{n}^{m}(\mathbf{r}) I_{n}^{m}(\rho),
$$

where the $g_{n}^{m, q}$ are unknown scalar constants. As mentioned before, the expansion is truncated at $n=3$ in order to use only the ellipsoidal harmonics that are analytically known. Using these equations, the scattered field can be represented as follows:

$$
\mathbf{H}_{3}^{s}(\mathbf{r})=\sum_{q=1}^{3} \frac{M_{q}}{4 \pi} \sum_{n=0}^{3} \sum_{m=1}^{2 n+1} g_{n}^{m, q}(2 n+1) \nabla\left[\mathbb{E}_{n}^{m}(\mathbf{r}) I_{n}^{m}(\rho)\right]
$$

The scalar constants $g_{n}^{m, q}$ have to be found by fitting the scalar boundary conditions given in equation (7) by the total field $\mathbf{H}_{3}^{t}=\mathbf{H}_{3}^{i}+\mathbf{H}_{3}^{s}$ on the surface $S$ of the ellipsoid. This surface is defined in the ellipsoidal coordinate system by the ellipsoidal surface $\rho=a_{1}$ for all $(\mu, \nu)$ of the ellipsoid of semi-axes $\left(a_{1}, a_{2}, a_{3}\right)$, while the normal $\mathbf{n}$ to this surface is the unit vector $\boldsymbol{\rho}$. This boundary equation $\left.\mathbf{n} \cdot \mathbf{H}_{3}^{t}(\mathbf{r})\right|_{S}=0$ is equivalent to

$$
\boldsymbol{\rho} \cdot\left\{\sum_{n=1}^{3} \sum_{m=1}^{2 n+1} g_{n}^{m, q}(2 n+1) \nabla\left[I_{n}^{m}(\rho) \mathbb{E}_{n}^{m}(\mathbf{r})\right]-\frac{2}{3} \mathbf{x}_{q}\right\}_{\rho=a_{1}}=0 \quad \forall(\mu, \nu) .
$$

Using the expression of $\boldsymbol{\rho}$ in the Cartesian coordinate system in (11) and equation (14) of the gradient, boundary conditions become

$$
\begin{aligned}
\left\{\sum_{n=1}^{3} \sum_{m=1}^{2 n+1} g_{n}^{m, q}\right. & \frac{(2 n+1)}{h_{\rho}}\left[\frac{d I_{n}^{m}(\rho)}{d \rho} E_{n}^{m}(\rho)+I_{n}^{m}(\rho) \frac{d E_{n}^{m}(\rho)}{d \rho}\right] E_{n}^{m}(\mu) E_{n}^{m}(\nu) \\
- & \left.\frac{2}{3} \frac{\rho}{h_{\rho}} \frac{h_{q}}{h_{1} h_{2} h_{3}} \frac{E_{1}^{q}(\rho)}{\left(\rho^{2}-a_{1}^{2}+a_{q}^{2}\right)} E_{1}^{q}(\mu) E_{1}^{q}(\nu)\right\}_{\rho=a_{1}}=0 \quad \forall(\mu, \nu) .
\end{aligned}
$$

Following the above, dealing with the orthogonal property of ellipsoidal harmonics, one can find that all $g_{n}^{m, q}$ are zero except $g_{1}^{q, q}$ :

$$
g_{1}^{q, q}=\frac{2}{9} \frac{a_{1} a_{2} a_{3}}{\left(a_{1} a_{2} a_{3} I_{1}^{q}\left(a_{1}\right)-1\right)} \frac{h_{q}}{h_{1} h_{2} h_{3}},
$$

where $q$ corresponds to the $M_{q}$ component of the magnetic dipole source. Note that these constants only depend on the semi-axes and foci of the ellipsoid and on the Carlson's elliptical integral of second kind, $I_{1}^{q}$, which is the only quantity that has to be computed using a standard mathematical library.

If needed, the expression of the scattered field of order three can be deduced as

$$
\begin{aligned}
\mathbf{H}_{3}^{s}(\mathbf{r}) & =\sum_{q=1}^{3} \frac{M_{q}}{4 \pi} 3 g_{1}^{q, q} \nabla\left[\mathbb{E}_{1}^{q}(\mathbf{r}) I_{1}^{q}(\rho)\right] \\
& =\sum_{q=1}^{3} \frac{M_{q}}{4 \pi} 3 g_{1}^{q, q} \frac{h_{1} h_{2} h_{3}}{h_{q}}\left[\frac{\rho}{h_{\rho}}\left(\sum_{i=1}^{3} \frac{x_{i} \mathbf{x}_{i}}{\rho^{2}-a_{1}^{2}+a_{i}^{2}}\right) \frac{d I_{1}^{q}(\rho)}{d \rho} x_{q}+I_{1}^{q}(\rho) \mathbf{x}_{q}\right],
\end{aligned}
$$


where the derivatives of the elliptic integral are analytically known:

$$
\frac{d I_{1}^{q}(\rho)}{d \rho}=\frac{-1}{\left(\rho^{2}-a_{1}^{2}+a_{q}^{2}\right) \sqrt{\rho^{2}-h_{2}^{2}} \sqrt{\rho^{2}-h_{2}^{3}}} .
$$

The solution obtained is very simple due to the uniformity of the incident field and the nullity of the electric field of order three.

3.3. Solution of the second-order term. The second-order term is more complicated to deal with because magnetic and electric fields are both non-zero. They also depend upon the static field $\mathbf{H}_{0}$ ( $\mathbf{E}_{0}$ being zero) already found and known as an approximate expansion in terms of scalar external ellipsoidal harmonics $\mathbb{F}_{n}^{m}(\mathbf{r})$ up to order two (or the internal one $\mathbb{E}_{n}^{m}(\mathbf{r})$ using equation (50) $)$ :

$$
\mathbf{H}_{0}^{s}(\mathbf{r})=\sum_{q=1}^{3} \frac{M_{q}}{4 \pi} \sum_{n=0}^{2} \sum_{m=1}^{2 n+1}(2 n+1) f_{n}^{m, q} \nabla\left(I_{n}^{m}(\rho) \mathbb{E}_{n}^{m}(\mathbf{r})\right)=\sum_{q=1}^{3} \frac{M_{q}}{4 \pi} \nabla \psi_{0 q}(\mathbf{r}),
$$

where $f_{n}^{m, q}$ are known scalar constants and $\psi_{0 q}$ is a scalar potential (see Appendix B). The following equations summarize the scattered problem and come from equations (5) and (6):

$$
\begin{array}{lrr}
\mathbf{H}_{2}^{i}(\mathbf{r})=-\frac{\mathbf{M}}{4 \pi} \cdot\left(\frac{\mathbf{R R}}{R^{2}}+\overline{\overline{\mathrm{I}}}\right) \frac{1}{2 R} & \Delta \mathbf{H}_{2}^{*}(\mathbf{r})=\mathbf{H}_{0}^{*}(\mathbf{r}) & \nabla \cdot \mathbf{H}_{2}^{*}=0 \\
\mathbf{E}_{2}^{i}(\mathbf{r})=-\frac{1}{\sigma} \frac{\mathbf{M}}{4 \pi} \times \frac{\mathbf{R}}{R^{3}} & \mathbf{E}_{2}^{*}(\mathbf{r})=\frac{1}{\sigma} \nabla \times \mathbf{H}_{2}^{*}(\mathbf{r}) & \nabla \cdot \mathbf{E}_{2}^{*}=0
\end{array}(*=t, i, s) .
$$

The order-two magnetic field satisfies a Poisson's equation, and the order-two electric field can be deduced from the previous field, but the problem must be solved by matching boundary conditions (7) both for the electric and magnetic total fields as for any electromagnetic problem.

The field $\mathbf{H}_{2}^{s}$ must satisfy the Poisson's equation, so it can be written as a superposition of a particular solution $\mathbf{H}_{2}^{s, p}$ and a harmonic solution $\mathbf{H}_{2}^{s, h}$. Using identities $\Delta(u \mathbf{r})=$ $(\Delta u) \mathbf{r}+u(\Delta \mathbf{r})+2 \nabla u \cdot \nabla \mathbf{r}$ and $\Delta \mathbf{r}=\mathbf{0}$, and having in mind that $\Delta \psi_{0 q}=0$, the particular solution is

$$
\mathbf{H}_{2}^{s, p}(\mathbf{r})=\frac{\mathbf{r}}{2} \sum_{q=1}^{3} \frac{M_{q}}{4 \pi} \psi_{0 q}(\mathbf{r})=\frac{\mathbf{r}}{2} \sum_{q=1}^{3} \frac{M_{q}}{4 \pi} \sum_{n=0}^{2} \sum_{m=1}^{2 n+1}(2 n+1) f_{n}^{m, q} I_{n}^{m}(\rho) \mathbb{E}_{n}^{m}(\mathbf{r}) .
$$

Note that this particular solution is truncated at $n=2$, taking into account the solution for $\mathbf{H}_{0}^{s}$ in equation (72) of Appendix B where constants $f_{3}^{m, q}$ are all zero.

Generally the harmonic solution $\mathbf{H}_{2}^{s, h}$ is written as an expansion of scalar harmonics up to infinity $(n=0, \cdots, \infty)$, but here (for the same reason as previously stated), only the scalar ellipsoidal harmonics up to order $3\left(\mathbb{E}_{3}^{m}(\mathbf{r}), m=1, \cdots, 7\right)$ are used. Due to the fact that the particular solution only involves harmonics up to order two $\left(\nabla \mathbb{E}_{2}^{m}, m=1, \cdots, 5\right)$, the harmonic solution $\mathbf{H}_{2}^{s, h}$ must also be written as an expansion on ellipsoidal harmonics up to order 2:

$$
\mathbf{H}_{2}^{s, h}(\mathbf{r})=\sum_{n=0}^{2} \sum_{m=1}^{2 n+1} \mathbf{e}_{n}^{m} \mathbb{F}_{n}^{m}(\mathbf{r})=\sum_{n=0}^{2} \sum_{m=1}^{2 n+1} \mathbf{e}_{n}^{m}(2 n+1) I_{n}^{m}(\rho) \mathbb{E}_{n}^{m}(\mathbf{r}),
$$


where $\mathbf{e}_{n}^{m}$ are vector unknown constants.

As mentioned before, the order-two electric field is linked to the magnetic field by the equation $\mathbf{E}_{2}^{s}=\nabla \times \mathbf{H}_{2}^{s} / \sigma$ that provides the expression of $\mathbf{E}_{2}^{s}$ as a superposition of a particular solution $\mathbf{E}_{2}^{s, p}$ and a harmonic solution $\mathbf{E}_{2}^{s, h}$ :

$$
\begin{aligned}
& \mathbf{E}_{2}^{s, p}(\mathbf{r})=\sum_{n=0}^{2} \sum_{m=1}^{2 n+1} \frac{1}{\sigma}(2 n+1) \sum_{q=1}^{3} \frac{M_{q}}{4 \pi} \frac{f_{n}^{m, q}}{2} \nabla \times\left[\mathbf{r} I_{n}^{m}(\rho) \mathbb{E}_{n}^{m}(\mathbf{r})\right] \\
& \mathbf{E}_{2}^{s, h}(\mathbf{r})=\sum_{n=0}^{2} \sum_{m=1}^{2 n+1} \frac{1}{\sigma}(2 n+1) \nabla \times\left[\mathbf{e}_{n}^{m} I_{n}^{m}(\rho) \mathbb{E}_{n}^{m}(\mathbf{r})\right] .
\end{aligned}
$$

Dealing with such types of expressions for $\mathbf{H}_{2}^{s}$ and $\mathbf{E}_{2}^{s}$ to satisfy the boundary conditions yields a harmonic solution that cannot be obtained up to $n=2$ and ends at $n=1$, which results in an unsatisfactory numerical solution.

In order to take into account higher-order harmonics, a more suitable harmonic solution with additive gradient terms can be sought as

$$
\mathbf{H}_{2}^{s, h}(\mathbf{r})=\sum_{n=0}^{2} \sum_{m=1}^{2 n+1}(2 n+1)\left(\mathbf{e}_{n}^{m}+d_{n}^{m} \nabla\right) I_{n}^{m}(\rho) \mathbb{F}_{n}^{m}(\mathbf{r}),
$$

where $d_{n}^{m}$ are new unknown scalar constants. The derivatives of ellipsoidal harmonics add a new contribution to the harmonic solution that can now better combine derivatives of ellipsoidal harmonics in the incident field. Using the newest expression of $\mathbf{H}_{2}^{s, h}$, the harmonic $\mathbf{E}_{2}^{s, h}$ solution of the scattered electric field becomes

$$
\mathbf{E}_{2}^{s, h}(\mathbf{r})=\sum_{n=0}^{2} \sum_{m=1}^{2 n+1} \frac{1}{\sigma}(2 n+1) \nabla \times\left[\left(\mathbf{e}_{n}^{m}+d_{n}^{m} \nabla\right) I_{n}^{m}(\rho) \mathbb{E}_{n}^{m}(\mathbf{r})\right]
$$

The constants $\mathbf{e}_{n}^{m}, d_{n}^{m}$ now have to be found using boundary conditions (7) and equation (28) of the problem. First of all, boundary conditions for the total magnetic field at the surface of the ellipsoid are

$$
\mathbf{n} \cdot\left[\mathbf{H}_{2}^{i}(\mathbf{r})+\mathbf{H}_{2}^{s, p}(\mathbf{r})+\mathbf{H}_{2}^{s, h}(\mathbf{r})\right]_{S}=\boldsymbol{\rho} \cdot\left[\mathbf{H}_{2}^{i}(\mathbf{r})+\mathbf{H}_{2}^{s, p}(\mathbf{r})+\mathbf{H}_{2}^{s, h}(\mathbf{r})\right]_{\rho=a_{1}}=0 .
$$

It is equivalent to

$$
\begin{aligned}
l_{a_{1}}(\mu, \nu) \sum_{n=0}^{2} \sum_{m=1}^{2 n+1}\{ & {\left[\sum_{i=1}^{3} A_{n}^{m, i}+D_{n}^{m} d_{n}^{m}\right] E_{n}^{m}(\mu) E_{n}^{m}(\nu) } \\
& \left.+\sum_{i=1}^{3}\left(B_{n}^{m, i}+e_{n}^{m, i} C_{n}^{m, i}\right)\left[x_{i} \mathbb{E}_{n}^{m}(\mathbf{r})\right]_{\rho=a_{1}}\right\}=0 \quad \forall(\mu, \nu),
\end{aligned}
$$


where

$$
\begin{aligned}
& A_{n}^{m, i}=\frac{M_{i}}{2}\left[(2 n+1) f_{n}^{m, i} I_{n}^{m}\left(a_{1}\right) E_{n}^{m}\left(a_{1}\right)-\frac{\mathbb{F}_{n}^{m}\left(\mathbf{r}_{0}\right)}{(2 n+1) \gamma_{n}^{m}} \frac{E_{n}^{m \prime}\left(a_{1}\right)}{a_{1}} x_{0 i}\right], \\
& D_{n}^{m}=(2 n+1)\left[a_{2} a_{3} I_{n}^{m}\left(a_{1}\right) E_{n}^{m \prime}\left(a_{1}\right)-\frac{1}{E_{n}^{m}\left(a_{1}\right)}\right] \frac{4 \pi}{a_{1} a_{2} a_{3}}, \\
& B_{n}^{m, i}=\frac{M_{i}}{2} \frac{\mathbb{F}_{n}^{m}\left(\mathbf{r}_{0}\right)}{(2 n+1) \gamma_{n}^{m}}\left[\frac{E_{n}^{m \prime}\left(a_{1}\right)}{a_{1} E_{n}^{m}\left(a_{1}\right)}-\frac{1}{a_{i}^{2}}\right], \\
& C_{n}^{m, i}=4 \pi(2 n+1) \frac{I_{n}^{m}\left(a_{1}\right)}{a_{i}^{2}}, \\
& l_{\rho}(\mu, \nu)=\left[\left(\rho^{2}-\mu^{2}\right)\left(\rho^{2}-\nu^{2}\right)\right]^{-1 / 2}, \\
& e_{n}^{m, i}=\mathbf{e}_{n}^{m} \cdot \mathbf{x}_{i} .
\end{aligned}
$$

The orthogonality between ellipsoidal harmonics provides a system of 9 equations with 36 unknowns (9 of the $d_{n}^{m}$ kind and 27 of the $e_{n}^{m, p}$ one). Boundary conditions for the electric field are needed to obtain the missing equations for the number of unknowns involved. This condition is

$$
\begin{aligned}
\mathbf{n} \times\left[\mathbf{E}_{2}^{i}(\mathbf{r})+\mathbf{E}_{2}^{s, p}(\mathbf{r})+\mathbf{E}_{2}^{s, h}(\mathbf{r})\right]_{S} & \\
& =\frac{1}{\sigma} \boldsymbol{\rho} \times\left[\nabla \times\left(\mathbf{H}_{2}^{i}(\mathbf{r})+\mathbf{H}_{2}^{s, p}(\mathbf{r})+\mathbf{H}_{2}^{s, h}(\mathbf{r})\right)\right]_{\rho=a_{1}}=\mathbf{0 .} .
\end{aligned}
$$

As a result, one can prove that, for all $(\mu, \nu)$, it is equivalent to

$$
\begin{gathered}
\sum_{n=0}^{2} \sum_{m=1}^{2 n+1} \sum_{i=1}^{3}\left\{\frac{(2 n+1)}{a_{1} a_{2} a_{3}\left[E_{n}^{m}\left(a_{1}\right)\right]^{2}}\left[\left(8 \pi e_{n}^{m, i}+\sum_{p=1}^{3} M_{p} f_{n}^{m, p} x_{i}\right) \mathbb{E}_{n}^{m}(\mathbf{r})[\overline{\overline{\mathrm{I}}}-\boldsymbol{\rho} \boldsymbol{\rho}] \cdot \mathbf{x}_{i}\right]_{\rho=a_{1}}\right. \\
+\left[2 \frac{\mathbb{F}_{n}^{m}\left(\mathbf{r}_{0}\right)}{(2 n+1) \gamma_{n}^{m}} M_{i}-(2 n+1) I_{n}^{m}\left(a_{1}\right)\left(8 \pi e_{n}^{m, i}+\sum_{p=1}^{3} M_{p} f_{n}^{m, p} x_{i}\right)_{\rho=a_{1}}\right] \\
\left.\left[\frac{E_{n}^{m \prime}\left(a_{1}\right)}{a_{1}} E_{n}^{m}(\mu) E_{n}^{m}(\nu) \mathbf{x}_{i}-\frac{1}{a_{i}^{2}}\left(x_{i} \nabla \mathbb{E}_{n}^{m}(\mathbf{r})\right)_{\rho=a_{1}}\right]\right\}=\mathbf{0}
\end{gathered}
$$

The term $[\overline{\overline{\mathrm{I}}}-\boldsymbol{\rho} \boldsymbol{\rho}]$ cannot be written in terms of ellipsoidal harmonics, so no orthogonality rules between harmonics can be applied at this step, and the equation $\nabla \cdot \mathbf{H}_{2}^{s}(\mathbf{r})=0$ is 
necessary. This equation is also valid for $\rho=a_{1}$, and it gives

$$
\begin{aligned}
& \sum_{n=0}^{2} \sum_{m=1}^{2 n+1} \sum_{i=1}^{3} \frac{(2 n+1)}{\left[E_{n}^{m}\left(a_{1}\right)\right]^{2}} {\left[\left(8 \pi e_{n}^{m, i}+\sum_{p=1}^{3} M_{p} f_{n}^{m, p} x_{i}\right) l_{a_{1}}(\mu, \nu) \mathbb{E}_{n}^{m}(\mathbf{r})\left(\mathbf{x}_{i} \cdot \boldsymbol{\rho}\right)\right]_{\rho=a_{1}} } \\
&=\sum_{n=0}^{2} \sum_{m=1}^{2 n+1} \sum_{i=1}^{3}(2 n+1)\left\{3 M_{i} f_{n}^{m, i} I_{n}^{m}\left(a_{1}\right) E_{n}^{m}\left(a_{1}\right) E_{n}^{m}(\mu) E_{n}^{m}(\nu)\right. \\
&\left.+I_{n}^{m}\left(a_{1}\right)\left[\left(8 \pi e_{n}^{m, i}+\sum_{p=1}^{3} M_{p} f_{n}^{m, p} x_{i}\right) \mathbf{x}_{i} \cdot \nabla \mathbb{E}_{n}^{m}(\mathbf{r})\right]_{\rho=a_{1}}\right\} .
\end{aligned}
$$

Putting this result into equation (38) and taking only the $\mathbf{x}_{i}$ component provides the following equation, for all $(\mu, \nu)$ :

$$
\begin{aligned}
& {\left[\sum_{n=0}^{2} \sum_{m=1}^{2 n+1}\left(2 B_{n+}^{m, i} E_{n}^{m}\left(a_{1}\right)-D_{n}^{m} e_{n}^{m, i}\right) E_{n}^{m}(\mu) E_{n}^{m}(\nu)\right.} \\
& +\sum_{n=0}^{2} \sum_{m=1}^{2 n+1} \sum_{p=1}^{3}\left(2 B_{n-}^{m, p}+C_{n}^{m, p} e_{n}^{m, p}\right) x_{p} \mathbf{x}_{i} \cdot \nabla \mathbb{E}_{n}^{m}(\mathbf{r}) \\
& +\sum_{n=0}^{2} \sum_{m=1}^{2 n+1}\left(\sum_{p=1}^{3} M_{p} f_{n}^{m, p}\right) \frac{1}{2}\left(\frac{-D_{n}^{m} x_{i} \mathbb{E}_{n}^{m}(\mathbf{r})}{4 \pi E_{n}^{m}\left(a_{1}\right)}+(2 n+1) I_{n}^{m}\left(a_{1}\right) \mathbf{x}_{i} \cdot \nabla \mathbb{E}_{n}^{m}(\mathbf{r})\right) \\
& -12 \sum_{m=1}^{3}\left(\sum_{p=1}^{3} M_{p} f_{1}^{m, p}\right) I_{1}^{m}\left(a_{1}\right) \frac{h_{1} h_{2} h_{3}}{2 h_{m} a_{i}^{2}} x_{m} x_{i} \\
& -5 \sum_{m=1}^{5}\left(\sum_{p=1}^{3} M_{p} f_{2}^{m, p}\right) \frac{I_{2}^{m}\left(a_{1}\right)}{2 a_{i}^{2}}\left[3 x_{i} \mathbb{E}_{2}^{m}(\mathbf{r})+\mathbf{r} \cdot\left(x_{i} \nabla \mathbb{E}_{2}^{m}(\mathbf{r})\right)\right] \\
& \left.-\sum_{m=1}^{5} \sum_{p=1}^{3} C_{2}^{m, i} e_{2}^{m, p} \mathbf{x}_{p} \cdot\left(x_{i} \nabla \mathbb{E}_{2}^{m}(\mathbf{r})\right)\right]_{\rho=a_{1}}=0,
\end{aligned}
$$

where the new constants $B_{n+}^{m, p}$ and $B_{n-}^{m, p}$ are defined as

$$
B_{n}^{m, p}=B_{n+}^{m, p}+B_{n-}^{m, p} \quad \text { with }\left\{\begin{array}{l}
B_{n+}^{m, p}=\frac{M_{p}}{2} \frac{\mathbb{F}_{n}^{m}\left(\mathbf{r}_{0}\right)}{(2 n+1) \gamma_{n}^{m}} \frac{E_{n}^{m \prime}\left(a_{1}\right)}{a_{1} E_{n}^{m}\left(a_{1}\right)} \\
B_{n-}^{m, p}=-\frac{M_{p}}{2} \frac{\mathbb{F}_{n}^{m}\left(\mathbf{r}_{0}\right)}{(2 n+1) \gamma_{n}^{m}} \frac{1}{a_{p}^{2}}
\end{array}\right.
$$

In equation (40), all terms including coordinate $x_{i}$ and harmonics or derivatives of harmonics can now be expressed in terms of ellipsoidal harmonics. The property of orthogonality between ellipsoidal harmonics applied to equation (40) results in a complicated system of equations as is explained in Appendix C.

As expected, it is a system of 27 equations with 27 unknowns of $e_{n}^{m, p}$ kind. Boundary conditions on the electric field will provide the vector constants $\mathbf{e}_{n}^{m}$ while the ones on the 
magnetic field will give the $d_{n}^{m}$ constants. The solutions obtained are

$$
\begin{gathered}
e_{0}^{1, i}=\frac{h_{1} h_{2} h_{3}}{2 h_{i}} \frac{\mathbf{M} \cdot \mathbf{f}_{1}^{i}}{4 \pi} \quad(\text { for } i=1,2,3), \\
e_{1}^{i, i}=\frac{h_{i} a_{i}^{2} a_{1} a_{2} a_{3}}{15 h_{1} h_{2} h_{3}}\left[\frac{\prod_{l=1}^{3}\left(\Lambda-a_{l}^{2}\right)}{\Lambda-a_{i}^{2}} D_{2}^{1} \mathbf{f}_{2}^{1}+\frac{\prod_{l=1}^{3}\left(\Lambda^{\prime}-a_{l}^{2}\right)}{\Lambda^{\prime}-a_{i}^{2}} D_{2}^{2} \mathbf{f}_{2}^{2}\right] \cdot \frac{\mathbf{M}}{(4 \pi)^{2}}, \\
e_{1}^{i, p}=\frac{h_{1} h_{2} h_{3}}{3\left(h_{p}+h_{i}\right)}\left[a_{1} a_{2} a_{3}\left(a_{p}^{2}+a_{i}^{2}\right) I_{2}^{p+i}\left(a_{1}\right)-1\right] \frac{\mathbf{M} \cdot \mathbf{f}_{2}^{p+i}}{4 \pi} \quad(\text { if } i \neq p)
\end{gathered}
$$

for $i=1,2,3$ and $p=1,2,3$.

$$
\begin{gathered}
e_{2}^{i, p}=0 \quad(\text { for } i=1, \cdots, 5 \text { and } p=1,2,3), \\
d_{0}^{1}=a_{1} a_{2} a_{3} \sum_{i=1}^{3} \frac{h_{1} h_{2} h_{3}}{h_{i}} I_{1}^{i}\left(a_{1}\right) e_{1}^{i, i}, \\
d_{1}^{i}=\frac{-1}{D_{1}^{i}}\left\{\sum_{p=1}^{3} A_{1}^{i, p}+\frac{a_{i} h_{i}}{h_{2} h_{2} h_{3}}\left[C_{0}^{1, i} e_{0}^{1, i}+B_{0}^{1, i}+\sum_{p=1}^{3} \frac{\left(1-\delta_{i p}\right)\left(h_{1} h_{2} h_{3}\right)^{2} a_{p}^{2} B_{2}^{i+p, p}}{5 h_{i} h_{p}}\right.\right. \\
\left.\left.+\frac{2 \Lambda \prod_{l=1}^{3}\left(\Lambda-a_{l}^{2}\right) B_{2}^{1, i}}{5\left(\Lambda-a_{i}^{2}\right)}+\frac{2 \Lambda^{\prime} \prod_{l=1}^{3}\left(\Lambda^{\prime}-a_{l}^{2}\right) B_{2}^{2, i}}{5\left(\Lambda^{\prime}-a_{i}^{2}\right)}\right]\right\} \quad(\text { for } i=1,2,3), \\
d_{2}^{l}=\frac{-1}{D_{2}^{l}} \sum_{p=1}^{3}\left[A_{2}^{l, p}+(-1)^{l} \frac{h_{1} h_{2} h_{3}}{\left(\Lambda-\Lambda^{\prime}\right)} \frac{a_{p}^{2} C_{1}^{p, p}}{3 h_{p}\left(L-a_{p}^{2}\right)} e_{1}^{p, p}\right]
\end{gathered}
$$

for $l=1,2$ with $L=\Lambda$ if $l=1$ and $L=\Lambda^{\prime}$ if $l=2$.

$$
\begin{aligned}
d_{2}^{i+p}=-\frac{a_{i} a_{p}}{D_{2}^{p+i}\left(h_{1} h_{2} h_{3}\right)}\left[\left(h_{i} C_{1}^{p, i}+h_{p} C_{1}^{i, p}\right) e_{1}^{i, p}+\left(h_{i} B_{1}^{p, i}+h_{p} B_{1}^{i, p}\right)\right. \\
\left.+\sum_{m=1}^{3} \frac{h_{1} h_{2} h_{3}}{a_{i} a_{p}} A_{2}^{p+i, m}\right] \quad(\text { for } i=1,2,3 \text { and } p=1,2,3 \text { but } i \neq p),
\end{aligned}
$$

where the $\mathbf{f}_{n}^{m}=\sum_{i=1}^{3} f_{n}^{m, i} \mathbf{x}_{i}$ are the vector constants involved in the static field (see Appendix B).

The scattered fields $\left(\mathbf{H}_{2}^{s}, \mathbf{E}_{2}^{s}\right)$ are known as approximate solutions for two reasons: first, the expressions of the fields are truncated at $n=2$ and so the solution involves ellipsoidal harmonics only up to order $3\left(\mathbb{E}_{3}^{m}(\mathbf{r})\right)$; second, the constants $\mathbf{e}_{n}^{m}$ and $d_{n}^{m}$ found are approximate expressions due to the truncation applied on the boundary conditions.

4. Numerical results. The solution given here has been implemented and tested only for the magnetic field, which is the most useful quantity at low frequencies. 
The ellipsoid is at the center of the Cartesian coordinate system $\left(x_{1}, x_{2}, x_{3}\right)$ in a conducting surrounding medium of $10^{-5} \mathrm{~S} / \mathrm{m}$. Its semi-axes are $a_{1}=75 \mathrm{~m}, a_{2}=50 \mathrm{~m}$, $a_{3}=25 \mathrm{~m}$. This ellipsoid is illuminated by a vertical magnetic dipole of vector intensity $\mathbf{M}=M \mathbf{x}_{1}$ with $M=10^{4} \mathrm{Am}^{2}$ at $50 \mathrm{~Hz}$, located at $\left(x_{1}, x_{2}, x_{3}\right)=(200 \mathrm{~m}, 0,0)$. The magnetic field it scatters is measured along a vertical line (parallel to $\mathbf{x}_{1}$ ) from $x_{1}=-200$ $\mathrm{m}$ to $x_{1}=200 \mathrm{~m}$ at $x_{2}=200 \mathrm{~m}$ and $x_{3}=0$.

The analytical results given in this paper are compared (see Figure 2) to a numerical evaluation of the magnetic field scattered by the same ellipsoid obtained using a finiteelement (FE) code (thanks to H. Torte2). The three components, real and imaginary parts, of the scattered field are displayed. The low-frequency results are compared to the FE results which can be considered as exact. In order to prove that the ellipsoidal behavior is really modeled by the LF results, the exact field scattered by a sphere of the same volume is also provided.

First of all, the LF results fit the ellipsoidal results better than the spherical one and, as expected, the LF solution includes information about the ellipsoidal shape. Looking at the real part of the fields, it can be noticed that the static term is sufficient, but for the imaginary part the order-two term $\mathbf{H}_{2}(j k)^{2}$ is needed because the static one is zero. The order-three term does not change the results significantly. This is due to the fact that at low frequency the value of $(j k)^{2}$ is nearly in $j \mathbb{R}$, while $(j k)^{3}$ has non-zero real and imaginary parts. For these reasons, the static term $\mathbf{H}_{0}$ and the $\mathbf{H}_{2}(j k)^{2}$ term will respectively provide the real or the imaginary part of the field, while $\mathbf{H}_{3}(j k)^{3}$ will contribute to both the real and the imaginary parts. This order-three term can be seen as an additive contribution that increases with frequency. To understand all of this, one should remember that for low-frequency expansion of a complex function $\psi=\sum \psi_{n}(\mathrm{j} k)^{n}$, functions $\psi_{n}$ are real.

Now, if one compares the LF and FE results for the ellipsoid, one can see a shift between results. Dealing with a low-frequency code for the sphere, it is possible to conclude that the problem lies in the number of harmonics used (up to $\mathbb{E}_{3}$ ). Here the truncation of the field at $n=2$ is not enough to take into account this shift. Harmonics $\mathbb{E}_{4}$ are needed but their analytical expressions in ellipsoidal geometry include constants that satisfy polynomial equations of order three which will result in a time-consuming solution. So, it is theoretically possible to find a new solution using harmonics $\mathbb{E}_{4}$ but the advantage of an inverse scheme will have to be estimated. Because the approximation given here provides very well the main behavior of the fields, it is not trivial that the analytical work with harmonics $\mathbb{E}_{4}$ is really interesting.

5. Conclusion. One of the main goals of the present investigation is to obtain an analytical approximation of the low-frequency behavior of the magnetic field scattered by a perfectly conducting ellipsoid illuminated by a magnetic dipole. The approximation obtained is a low-frequency expansion of the electric and magnetic scattered fields. The static term provides a very good approximation of the real part of the magnetic field, while the order-two term (the first non-zero term) contributes to the main behavior of the

${ }^{2}$ Hervé Tortel, Associate professor, Institut Fresnel, Marseille, France. 

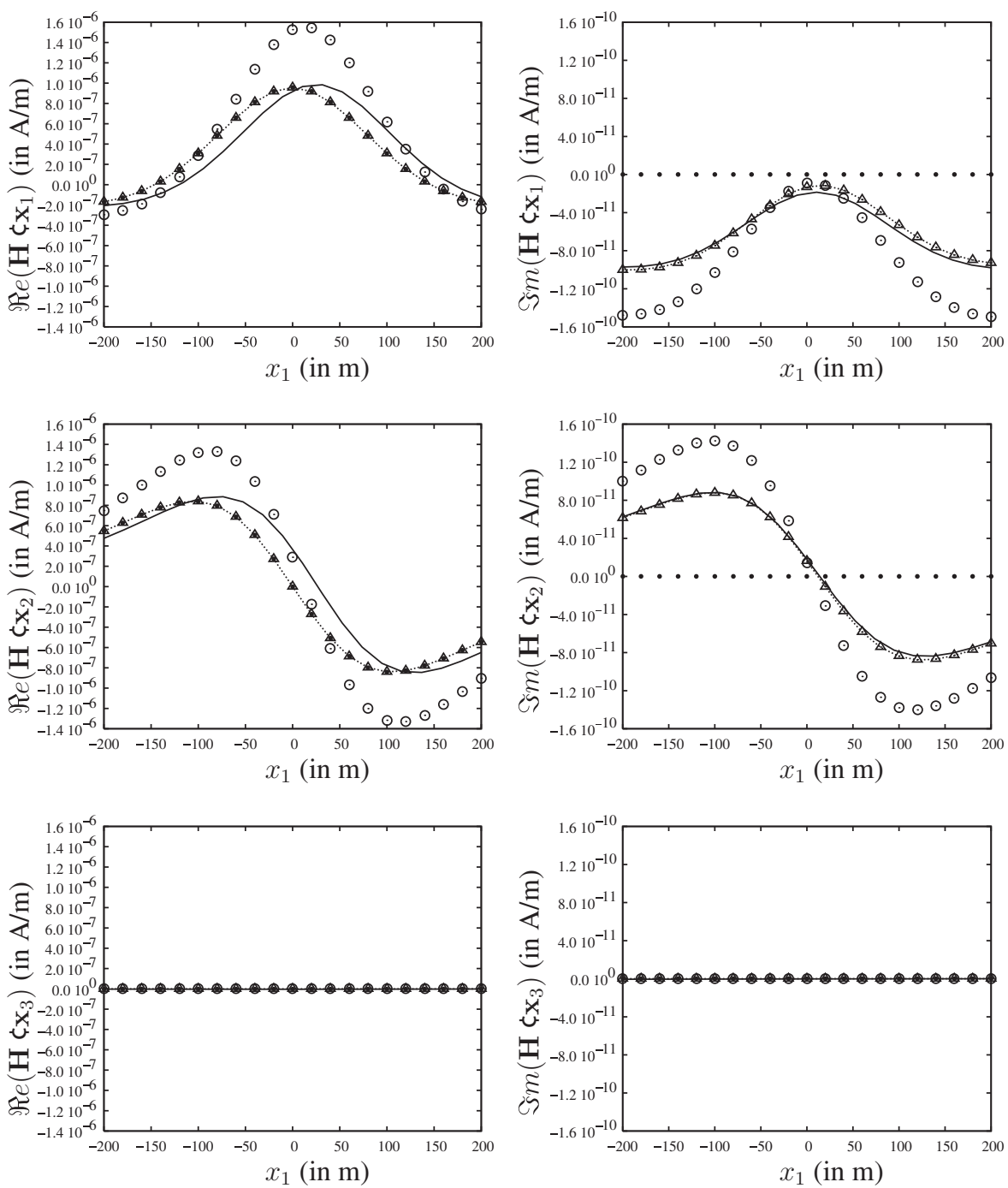

FIG. 2. Real and imaginary parts of the exact magnetic field scattered by a sphere (o), of the approximated LF one $\mathbf{H}_{0}(\bullet), \mathbf{H}_{0}+$ $\mathbf{H}_{2}(\mathrm{j} k)^{2}(\cdots), \mathbf{H}_{0}+\mathbf{H}_{2}(\mathrm{j} k)^{2}+\mathbf{H}_{3}(\mathrm{j} k)^{3}(\Delta)$ and of the FE one $(-)$ scattered by an ellipsoid

imaginary part, the order-three term being a correction to both the real and imaginary parts.

The solution given here appears to be a good approximation at low frequencies and suitably describes the ellipsoidal shape behavior. The advantages of the formulation lie in the analytical expressions that are algebraic ones, involving simple analytically-known constants. Consequently, the numerical evaluations of the fields are very fast, which is the main point of this work. This can be very useful for inverse schemes for localization and identification of buried objects at low frequencies. 
The need of the order-two term is also due to such applications. The identification of an unknown scatterer can be better achieved if the field is better described. The information contained in the imaginary part (given by the order-two term) that is smaller than the real part might help the inverse scheme to separate between different solutions of ellipsoidal parameters (orientation, size, $\cdots$ ).

The need of higher-order harmonics at low frequency is not clear. It will certainly provide a better fit for the real part, which is the more important one, but will it be useful for the inverse scheme knowing that it certainly will be more time consuming?

Appendix A. Ellipsoidal harmonics. Here, some details about ellipsoidal harmonics are given in terms of either Cartesian coordinates or ellipsoidal coordinates.

External $\mathbb{F}_{n}^{m}(\mathbf{r})$ and internal $\mathbb{E}_{n}^{m}(\mathbf{r})$ ellipsoidal harmonics are defined for $n \in \mathbb{N}$ and $m=1, \cdots, 2 n+1$. They are linked together by the relation

$$
\mathbb{F}_{n}^{m}(\mathbf{r})=(2 n+1) I_{n}^{m}(\rho) \mathbb{E}_{n}^{m}(\mathbf{r})
$$

where $\rho$ (defined for $\rho^{2} \geq h_{2}^{2}$ ) is one of the ellipsoidal coordinates of point $\mathbf{r}$ and $I_{n}^{m}$ is a Carlson's incomplete elliptic integral of $(n+1)$ th kind. Ellipsoidal harmonics are separated variable functions in the ellipsoidal coordinate, so they can be written as

$$
\mathbb{F}_{n}^{m}(\mathbf{r})=F_{n}^{m}(\rho) E_{n}^{m}(\mu) E_{n}^{m}(\mu), \quad \mathbb{E}_{n}^{m}(\mathbf{r})=E_{n}^{m}(\rho) E_{n}^{m}(\mu) E_{n}^{m}(\mu)
$$

with $F_{n}^{m}(\rho)=(2 n+1) E_{n}^{m}(\rho)$ and $\mathbf{r}=(\rho, \mu, \nu)$ as defined in Section 3.1. The elliptic integral $I_{n}^{m}(\rho)$ is defined in terms of $E_{n}^{m}(u)$ by

$$
I_{n}^{m}(\rho)=\int_{\rho}^{\infty} \frac{d u}{\left[E_{n}^{m}(u)\right]^{2} \sqrt{u^{2}-h_{3}^{2}} \sqrt{u^{2}-h_{2}^{2}}} .
$$

The first four ellipsoidal harmonics are known in the ellipsoidal coordinate system for which only the separated function $E_{n}^{m}(u)$ is needed. The $u$ variable can be $\rho, \mu$ or $\nu$, while keeping in mind that ellipsoidal coordinates are defined for $\rho^{2} \geq h_{2}^{2} \geq \mu^{2} \geq h_{3}^{2} \geq \nu^{2} \geq 0$.

$$
\begin{aligned}
& E_{0}^{1}(u)=1, \\
& E_{1}^{m}(u)=\sqrt{\left|u^{2}-a_{1}^{2}+a_{m}^{2}\right|} \quad(m=1,2,3), \\
& E_{2}^{l}(u)=u^{2}-a_{1}^{2}+L \quad(l, L)=(1, \Lambda) \text { or }\left(2, \Lambda^{\prime}\right), \\
& E_{2}^{6-m}(u)=\frac{\prod_{i=1}^{3} \sqrt{\left|u^{2}-a_{1}^{2}+a_{i}^{2}\right|}}{\sqrt{\left|u^{2}-a_{1}^{2}+a_{m}^{2}\right|}} \quad(m=1,2,3), \\
& E_{3}^{2 m-1}(u)=\sqrt{\left|u^{2}-a_{1}^{2}+a_{m}^{2}\right|}\left(u^{2}-a_{1}^{2}+\Lambda_{m}\right) \quad(m=1,2,3), \\
& E_{3}^{2 m}(u)=\sqrt{\left|u^{2}-a_{1}^{2}+a_{m}^{2}\right|}\left(u^{2}-a_{1}^{2}+\Lambda_{m}^{\prime}\right) \quad(m=1,2,3), \\
& E_{3}^{7}(u)=\prod_{i=1}^{3} \sqrt{\left|u^{2}-a_{1}^{2}+a_{i}^{2}\right|},
\end{aligned}
$$


where $\left(\Lambda, \Lambda^{\prime}\right)$ and $\left(\Lambda_{q}, \Lambda_{q}^{\prime}\right)$ respectively are solutions of the second-order polynomial equations below:

$$
\sum_{i=1}^{3} \frac{1}{\Lambda-a_{i}^{2}}=\sum_{i=1}^{3} \frac{1}{\Lambda^{\prime}-a_{i}^{2}}=0, \quad \sum_{i=1}^{3} \frac{1+2 \delta_{i q}}{\Lambda_{p}-a_{i}^{2}}=\sum_{i=1}^{3} \frac{1+2 \delta_{i q}}{\Lambda_{p}^{\prime}-a_{i}^{2}}=0(\text { for } p=1,2,3)
$$

( $\delta_{i q}$ being the Kronecker delta function). Their analytical expressions are simple and easy to find:

$$
\begin{aligned}
& \left.\begin{array}{l}
\Lambda \\
\Lambda^{\prime}
\end{array}\right\}=\frac{1}{3}\left[\left(3 a_{1}^{2}-h_{2}^{2}-h_{3}^{2}\right) \pm \sqrt{h_{1}^{4}+h_{2}^{2} h_{3}^{2}}\right], \\
& \left.\begin{array}{l}
\Lambda_{p} \\
\Lambda_{p}^{\prime}
\end{array}\right\}=\frac{1}{5}\left[\left(6 a_{1}^{2}-a_{p}^{2}-2 h_{2}^{2}-2 h_{3}^{2}\right) \pm \sqrt{4 h_{p}^{4}-(-1)^{p} \frac{\left(h_{1} h_{2} h_{3}\right)^{2}}{h_{p}^{2}}}\right] \quad(p=1,2,3) .
\end{aligned}
$$

The first four internal ellipsoidal harmonics are also known in a simple analytical form in the Cartesian coordinate system for $\mathbf{r}=\left(x_{1}, x_{2}, x_{3}\right)$ :

$$
\begin{aligned}
& \mathbb{E}_{0}^{1}(\mathbf{r})=1, \\
& \mathbb{E}_{1}^{m}(\mathbf{r})=h_{1} h_{2} h_{3} \frac{x_{m}}{h_{m}} \quad(m=1,2,3), \\
& \mathbb{E}_{2}^{l}(\mathbf{r})=\left(L-a_{1}^{2}\right)\left(L-a_{2}^{2}\right)\left(L-a_{3}^{2}\right)\left[\sum_{i=1}^{3} \frac{x_{i}^{2}}{L-a_{i}^{2}}+1\right] \quad(l, L)=(1, \Lambda) \text { or }\left(2, \Lambda^{\prime}\right), \\
& \mathbb{E}_{2}^{6-m}(\mathbf{r})=h_{1} h_{2} h_{3} x_{1} x_{2} x_{3} \frac{h_{m}}{x_{m}} \quad(m=1,2,3), \\
& \mathbb{E}_{3}^{2 m-1}(\mathbf{r})=h_{1} h_{2} h_{3}\left(\prod_{i=1}^{3}\left(\Lambda_{m}-a_{i}^{2}\right)\right) \frac{x_{m}}{h_{m}}\left[\sum_{p=1}^{3} \frac{x_{p}^{2}}{\Lambda_{m}-a_{p}^{2}}+1\right] \quad(m=1,2,3), \\
& \mathbb{E}_{3}^{2 m}(\mathbf{r})=h_{1} h_{2} h_{3}\left(\prod_{i=1}^{3}\left(\Lambda_{m}^{\prime}-a_{i}^{2}\right)\right) \frac{x_{m}}{h_{m}}\left[\sum_{p=1}^{3} \frac{x_{p}^{2}}{\Lambda_{m}^{\prime}-a_{p}^{2}}+1\right] \quad(m=1,2,3), \\
& \mathbb{E}_{3}^{7}(\mathbf{r})=\left(h_{1} h_{2} h_{3}\right)^{2} x_{1} x_{2} x_{3} .
\end{aligned}
$$

The expansion of the inverse distance on ellipsoidal harmonics is given in (15) but rewritten here for better understanding:

$$
\frac{1}{\left|\mathbf{r}-\mathbf{r}_{0}\right|}=\sum_{n=0}^{\infty} \sum_{m=1}^{2 n+1} \frac{1}{(2 n+1) \gamma_{n}^{m}} \mathbb{F}_{n}^{m}\left(\mathbf{r}_{0}\right) \mathbb{E}_{n}^{m}(\mathbf{r}) \quad \text { for } \rho<\rho_{0}
$$

In this expression the $\gamma_{n}^{m}$ constants are defined by

$$
\gamma_{n}^{m}=\frac{1}{4 \pi} \oint_{S\left(\rho=a_{1}\right)} \frac{\left(E_{n}^{m}(\mu) E_{n}^{m}(\nu)\right)^{2}}{\sqrt{a_{1}^{2}-\mu^{2}} \sqrt{a_{1}^{2}-\nu^{2}}} d S(\mu, \nu) .
$$


Focusing on the establishment of analytical formulations, their algebraic forms are used and the known ones are listed below:

$$
\begin{aligned}
& \gamma_{0}^{1}=1, \\
& \gamma_{1}^{m}=\frac{h_{1}^{2} h_{2}^{2} h_{3}^{2}}{3 h_{m}^{2}} \quad(\text { for } m=1,2,3), \\
& \gamma_{2}^{l}=\frac{(-)^{l} 2}{5}\left(\Lambda-\Lambda^{\prime}\right)\left(L-a_{1}^{2}\right)\left(L-a_{2}^{2}\right)\left(L-a_{3}^{2}\right) \quad \text { for }(l, L)=(1, \Lambda) \text { or }\left(2, \Lambda^{\prime}\right), \\
& \gamma_{2}^{6-m}=\frac{h_{m}^{2}}{15} h_{1}^{2} h_{2}^{2} h_{3}^{2} \quad(\text { for } m=1,2,3), \\
& \left.\gamma_{3}^{2 m-1}=\frac{-2}{21} \frac{h_{1}^{2} h_{2}^{2} h_{3}^{2}}{h_{m}^{2}}\left(\Lambda_{m}-\Lambda_{m}^{\prime}\right)\left(\prod_{l=1}^{3}\left(\Lambda_{m}-a_{l}^{2}\right)\right) \quad \quad \quad \text { (for } m=1,2,3\right), \\
& \gamma_{3}^{2 m}=\frac{2}{21} \frac{h_{1}^{2} h_{2}^{2} h_{3}^{2}}{h_{m}^{2}}\left(\Lambda_{m}-\Lambda_{m}^{\prime}\right)\left(\prod_{l=1}^{3}\left(\Lambda_{m}^{\prime}-a_{l}^{2}\right)\right) \quad(\text { for } m=1,2,3), \\
& \gamma_{3}^{7}=\frac{1}{105} h_{1}^{4} h_{2}^{4} h_{3}^{4} .
\end{aligned}
$$

Appendix B. Static solution. In the following, detailed calculations for the static fields, also called order-zero fields in our low-frequency approximation, are described. The static electric field $\mathbf{E}_{0}^{*}$ is zero for our problem (see equation (6) ), so that the static magnetic field is both curl and div free:

$$
\mathbf{E}_{0}^{*}(\mathbf{r})=\mathbf{0} \quad \nabla \times \mathbf{H}_{0}^{*}(\mathbf{r})=\mathbf{0} \quad \nabla \cdot \mathbf{H}_{0}^{*}(\mathbf{r})=0,
$$

where $*=i, t, s$ stands for the incident, total or scattered field, respectively. The total magnetic field satisfies the boundary conditions on the surface $S$ of the perfectly conductive ellipsoid, which consists of the cancellation of the normal component of this field $\mathbf{n} \cdot \mathbf{H}_{0}^{t}(\mathbf{r})=0$ at $\rho=a_{1}$ for all $(\mu, \nu)$. One can easily recognize a problem of potential where the static magnetic field is the gradient of a scalar potential:

$$
\Delta \psi_{0}(\mathbf{r})=0 \quad \mathbf{H}_{0}^{s}(\mathbf{r})=\nabla \psi_{0}(\mathbf{r}) .
$$

The source is a vector magnetic dipole $\mathbf{M}$ with general orientation. In the Cartesian coordinate system it can be written as the superposition of three magnetic dipoles oriented along the Cartesian axes $\mathbf{M}=\sum_{q=1}^{3} M_{q} \mathbf{x}_{q}$. Using the property of superposition, the static magnetic field can be viewed as a superposition of gradients of three scalar potentials corresponding to each dipole:

$$
\mathbf{H}_{0}^{s}(\mathbf{r})=\sum_{q=1}^{3} \frac{M_{q}}{4 \pi} \nabla \psi_{0 q}(\mathbf{r}) .
$$

For convenience the constant $4 \pi$ is extracted from the potentials.

The static incident magnetic field radiated by the magnetic dipole $\mathbf{M}$ is

$$
\mathbf{H}_{0}^{i}(\mathbf{r})=\frac{\mathbf{M}}{4 \pi} \cdot\left(\frac{3 \mathbf{R R}}{R^{2}}-\overline{\overline{\mathrm{I}}}\right) \frac{1}{R^{3}},
$$


where $1 / \mathbf{R}$ expansion in terms of the first four known ellipsoidal harmonics is

$$
\frac{1}{\left|\mathbf{r}-\mathbf{r}_{0}\right|}=\sum_{n=0}^{3} \sum_{m=1}^{2 n+1} \frac{1}{(2 n+1) \gamma_{n}^{m}} \mathbb{F}_{n}^{m}\left(\mathbf{r}_{0}\right) \mathbb{E}_{n}^{m}(\mathbf{r}) \quad \text { for } \rho<\rho_{0}
$$

This expansion is only needed for $\rho<\rho_{0}$ because the incident field is only used in the boundary conditions.

Potentials involved in the scattered magnetic field have a similar expansion in terms of exterior ellipsoidal harmonics:

$$
\psi_{0 q}(\mathbf{r})=\sum_{n=0}^{3} \sum_{m=1}^{2 n+1} f_{n}^{m, q} \mathbb{F}_{n}^{m}(\mathbf{r})=\sum_{n=0}^{3} \sum_{m=1}^{2 n+1}(2 n+1) f_{n}^{m, q} \mathbb{E}_{n}^{m}(\mathbf{r}) I_{n}^{m}(\rho)
$$

$f_{n}^{m, q}$ are unknown constants that have to be found using appropriate boundary conditions given above and rewritten below. For this, one uses expressions of the incident and scattered fields in (63) and (62), knowing that the normal vector $\mathbf{n}$ at the ellipsoidal surface $\left(S\left(\rho=a_{1}\right)\right)$ is the unit vector $\boldsymbol{\rho}$.

$$
\begin{aligned}
\left.\mathbf{n} \cdot \mathbf{H}_{0}^{t}(\mathbf{r})\right|_{S} & =\sum_{q=1}^{3} \frac{M_{q}}{4 \pi} \boldsymbol{\rho} \cdot\left[\nabla \nabla \frac{1}{R} \cdot \mathbf{x}_{q}+\nabla \psi_{0 q}(\mathbf{r})\right]_{\rho=a_{1}} \\
& =\sum_{q=1}^{3} \frac{M_{q}}{4 \pi} \frac{1}{h_{\rho}} \frac{\partial}{\partial \rho}\left[\frac{\partial}{\partial x_{q}} \frac{1}{R}+\psi_{0 q}(\mathbf{r})\right]_{\rho=a_{1}}=0 \quad \forall(\mu, \nu) .
\end{aligned}
$$

This equation, written in term of partial derivatives, is equivalent to the next equation for the $M_{q} \mathbf{x}_{q}$ illumination with $q=1,2,3$ :

$$
\begin{aligned}
\sum_{n=0}^{3} \sum_{m=1}^{2 n+1} \frac{1}{h_{\rho}} \frac{\partial}{\partial \rho}\left[\frac{\mathbb{F}_{n}^{m}\left(\mathbf{r}_{0}\right)}{(2 n+1) \gamma_{n}^{m}} \frac{\partial \mathbb{E}_{n}^{m}(\mathbf{r})}{\partial x_{q}}\right. & \\
\left.+(2 n+1) f_{n}^{m, q} \mathbb{E}_{n}^{m}(\mathbf{r}) I_{n}^{m}(\rho)\right]_{\rho=a_{1}} & =0 \quad \forall(\mu, \nu) .
\end{aligned}
$$

It appears that the derivatives of the ellipsoidal harmonics involved have to be calculated before applying the orthogonality properties between them. The derivatives are not given here but they can be easily calculated using the expression of ellipsoidal harmonics and 
the constants they contain. At the end, one obtains the constants, for $i=1,2,3$ :

$$
\begin{gathered}
f_{0}^{1, i}=\frac{a_{1} a_{2} a_{3} h_{i}}{h_{1} h_{2} h_{3}\left(\Lambda_{i}-\Lambda_{i}^{\prime}\right)} \sum_{p=1}^{3}\left(1+2 \delta_{p i}\right)\left[\frac{\mathbb{F}_{3}^{2 i}\left(\mathbf{r}_{0}\right)}{\left(\Lambda_{i}^{\prime}-a_{p}^{2}\right)}-\frac{\mathbb{F}_{3}^{2 i-1}\left(\mathbf{r}_{0}\right)}{\left(\Lambda_{i}-a_{p}^{2}\right)}\right]=0 \\
f_{1}^{m, i}=\frac{a_{1} a_{2} a_{3}}{1-a_{1} a_{2} a_{3} I_{1}^{m}\left(a_{1}\right)} \frac{h_{m}^{2} h_{i}}{\left(h_{1} h_{2} h_{3}\right)^{3}}\left[\mathbb{F}_{2}^{m+i}\left(\mathbf{r}_{0}\right)\left(1-\delta_{m i}\right)\right. \\
\left.+\frac{\left(\Lambda-a_{i}^{2}\right) \mathbb{F}_{2}^{2}\left(\mathbf{r}_{0}\right)-\left(\Lambda^{\prime}-a_{i}^{2}\right) \mathbb{F}_{2}^{1}\left(\mathbf{r}_{0}\right)}{\left(\Lambda-\Lambda^{\prime}\right)} \delta_{m i}(-1)^{i+1}\right] \quad(\text { for } m=1,2,3), \\
f_{2}^{l, i}=\frac{(-1)^{l+1} 3 L a_{1} a_{2} a_{3}}{5\left[1-2 L a_{1} a_{2} a_{3} I_{2}^{l}\left(a_{1}\right)\right]} \frac{h_{i}}{\left(h_{1} h_{2} h_{3}\right)^{3}} \frac{1}{\left(\Lambda-\Lambda^{\prime}\right)\left(\Lambda_{i}-\Lambda_{i}^{\prime}\right)} \\
\quad \times \sum_{p=1}^{3}(-1)^{p}\left(1+2 \delta_{p i}\right) h_{p}^{2}\left(L^{*}-a_{p}^{2}\right)\left[\frac{\mathbb{F}_{3}^{2 i}\left(\mathbf{r}_{0}\right)}{\left(\Lambda_{i}^{\prime}-a_{p}^{2}\right)}-\frac{\mathbb{F}_{3}^{2 i-1}\left(\mathbf{r}_{0}\right)}{\left(\Lambda_{i}-a_{p}^{2}\right)}\right] \\
f_{2}^{6-m, i}=\frac{3\left(2-\delta_{i m}\right)\left(\sum_{p=1}^{3} a_{p}^{2}-a_{m}^{2}\right) a_{1} a_{2} a_{3}}{5\left[1-\left(\sum_{p=1}^{3} a_{p}^{2}-a_{m}^{2}\right) a_{1} a_{2} a_{3} I_{2}^{6-m}\left(a_{1}\right)\right]} \frac{h_{i}}{h_{m}^{2} h_{1} h_{2} h_{3}} \\
\times\left[\frac{\left(1-\delta_{m i}\right)}{2 h_{i}^{2}\left(\Lambda_{6-(m+i)}-\Lambda_{6-(m+i)}^{\prime}\right)}\left(\frac{\mathbb{F}_{3}^{2(6-(m+i))}\left(\mathbf{r}_{0}\right)}{\left(\Lambda_{6-(m+i)}^{\prime}-a_{i}^{2}\right)}-\frac{\mathbb{F}_{3}^{2(6-(m+i))-1}\left(\mathbf{r}_{0}\right)}{\left(\Lambda_{6-(m+i)}-a_{i}^{2}\right)}\right)\right. \\
\left.+\frac{5}{\left(h_{1} h_{2} h_{3}\right)^{2}} \mathbb{F}_{3}^{7}\left(\mathbf{r}_{0}\right) \delta_{m i}\right] \quad(\text { for } m=1,2,3)
\end{gathered}
$$

$f_{3}^{m, i}$ being zero for $m=1, \cdots, 7$ and $i=1,2,3$.

At this step, the scattered static field is known in an approximate form due to the limited number of harmonics used. It can be written in terms of the ellipsoidal coordinate $\rho$, and the Cartesian ones $\left(x_{1}, x_{2}, x_{3}\right)$ along the unit Cartesian vectors $\left(\mathbf{x}_{1}, \mathbf{x}_{2}, \mathbf{x}_{3}\right)$ :

$$
\begin{aligned}
\mathbf{H}_{0}^{s}(\mathbf{r})=\sum_{q=1}^{3} \frac{M_{q}}{4 \pi} \sum_{n=0}^{3} \sum_{m=1}^{2 n+1} f_{n}^{m, q}(2 n+1) & {\left[I_{n}^{m}(\rho) \sum_{i=1}^{3} \frac{\partial \mathbb{E}_{n}^{m}(\mathbf{r})}{\partial x_{i}} \mathbf{x}_{i}\right.} \\
& \left.-\frac{\mathbb{E}_{n}^{m}(\mathbf{r})\left[\mathbb{E}_{n}^{m}(\rho)\right]^{-2}}{\sqrt{\rho-h_{2}^{2}} \sqrt{\rho-h_{3}^{2}}} \frac{\rho}{h_{\rho}^{2}} \sum_{i=1}^{3} \frac{x_{i} \mathbf{x}_{i}}{\left(\rho^{2}-a_{1}^{2}+a_{i}^{2}\right)}\right] .
\end{aligned}
$$

The derivatives involved here, as mentioned before, can be calculated in an analytical form in the Cartesian coordinate system. This result is finally quite simple and one can compute the scattered field in any Cartesian coordinate system.

Appendix C. Solution for the order-two fields. In this appendix, only some details are given in order to explain some long but easy points of the main work of Section 3.3. So the general framework is not examined again. 
From the first boundary conditions

$$
\begin{aligned}
& \sum_{n=0}^{2} \sum_{m=1}^{2 n+1}\left\{\left[\sum_{i=1}^{3} A_{n}^{m, i}+D_{n}^{m} d_{n}^{m}\right] E_{n}^{m}(\mu) E_{n}^{m}(\nu)\right. \\
&\left.\quad+\sum_{i=1}^{3}\left(B_{n}^{m, i}+e_{n}^{m, i} C_{n}^{m, i}\right)\left[x_{i} \mathbb{E}_{n}^{m}(\mathbf{r})\right]_{\rho=a_{1}}\right\}=0 \quad \forall(\mu, \nu),
\end{aligned}
$$

where $A_{n}^{m, i}, B_{n}^{m, i}, C_{n}^{m, i}$ and $D_{n}^{m}$ are known constants, the unknown constants $e_{n}^{m, i}$ and $d_{n}^{m}$ have to be determined. Terms $x_{i} \mathbb{E}_{n}^{m}(\mathbf{r})$ must be rewritten in terms of other internal ellipsoidal harmonics before applying the orthogonal property of harmonics. The term with harmonics $E_{0}^{1}(\mu) E_{0}^{1}(\nu)$ provides equation

$$
D_{0}^{1} d_{0}^{1}+\sum_{i=1}^{3}\left[A_{0}^{1, i}+\frac{h_{1} h_{2} h_{3}}{h_{i}} \frac{a_{i}^{2}}{3}\left(B_{1}^{i, i}+C_{1}^{i, i} e_{1}^{i, i}\right)\right]=0
$$

where $A_{0}^{1, i}=B_{1}^{i, i}=0$, while the one with $E_{1}^{i}(\mu) E_{1}^{i}(\nu)$ (for $\left.i=1, \cdots, 3\right)$ results in

$$
\begin{gathered}
5\left(C_{0}^{1, i} e_{0}^{1, i}+B_{0}^{1, i}\right)+\frac{5 h_{1} h_{2} h_{3}}{a_{i} h_{i}}\left[\sum_{p=1}^{3} A_{1}^{i, p}+D_{1}^{i} d_{1}^{i}\right] \\
+\sum_{p=1}^{3} \frac{\left(1-\delta_{i p}\right)\left(h_{1} h_{2} h_{3}\right)^{2} a_{p}^{2}\left(C_{2}^{i+p, p} e_{2}^{i+p, p}+B_{2}^{i+p, p}\right)}{h_{i} h_{p}} \\
+\frac{2 \Lambda \prod_{l=1}^{3}\left(\Lambda-a_{l}^{2}\right)\left(C_{2}^{1, i} e_{2}^{1, i}+B_{2}^{1, i}\right)}{\Lambda-a_{i}^{2}}+\frac{2 \Lambda^{\prime} \prod_{l=1}^{3}\left(\Lambda^{\prime}-a_{l}^{2}\right)\left(C_{2}^{2, i} e_{2}^{2, i}+B_{2}^{2, i}\right)}{\Lambda^{\prime}-a_{i}^{2}}=0 .
\end{gathered}
$$

The orthogonality for $E_{2}^{1}(\mu) E_{2}^{1}(\nu)$ and $E_{2}^{2}(\mu) E_{2}^{2}(\nu)$ gives similar equations summarized by

$$
\sum_{i=1}^{3}\left[A_{2}^{l, i}+\frac{h_{1} h_{2} h_{3}}{h_{i}} \frac{a_{i}^{2}}{3} \frac{(-1)^{l}}{\left(\Lambda-\Lambda^{\prime}\right)\left(L-a_{i}^{2}\right)}\left(B_{1}^{i, i}+C_{1}^{i, i} e_{1}^{i, i}\right)\right]+D_{2}^{l} d_{2}^{l}=0
$$

where $(l, L)$ is either $(1, \Lambda)$ or $\left(2, \Lambda^{\prime}\right)$. The harmonics $E_{2}^{i+m}(\mu) E_{2}^{i+m}(\nu)$ provide simple equations:

$$
\begin{gathered}
h_{i} C_{1}^{m, i} e_{1}^{m, i}+h_{m} C_{1}^{i, m} e_{1}^{i, m}+\left(h_{i} B_{1}^{m, i}+h_{m} B_{1}^{i, m}\right) \\
+\frac{h_{1} h_{2} h_{3}}{a_{i} a_{m}}\left(A_{2}^{i+m, i}+A_{2}^{i+m, m}+D_{2}^{m+i} d_{2}^{m+i}\right)=0 \\
\quad(i \neq m ; i=1, \cdots, 3 ; m=1, \cdots, 3) .
\end{gathered}
$$


The $x_{i}$ component of the second boundary equation linked to the divergence-free property of the magnetic field, as explained in Section 3.3, is

$$
\begin{aligned}
& {\left[\sum_{n=0}^{2} \sum_{m=1}^{2 n+1}\left(2 B_{n+}^{m, i} E_{n}^{m}\left(a_{1}\right)-D_{n}^{m} e_{n}^{m, i}\right) E_{n}^{m}(\mu) E_{n}^{m}(\nu)\right.} \\
& +\sum_{n=0}^{2} \sum_{m=1}^{2 n+1} \sum_{p=1}^{3}\left(2 B_{n-}^{m, p}+C_{n}^{m, p} e_{n}^{m, p}\right) x_{p} \mathbf{x}_{i} \cdot \nabla \mathbb{E}_{n}^{m}(\mathbf{r}) \\
& +\sum_{n=0}^{2} \sum_{m=1}^{2 n+1}\left(\sum_{p=1}^{3} M_{p} f_{n}^{m, p}\right) \frac{1}{2}\left(\frac{-D_{n}^{m} x_{i} \mathbb{E}_{n}^{m}(\mathbf{r})}{4 \pi E_{n}^{m}\left(a_{1}\right)}+(2 n+1) I_{n}^{m}\left(a_{1}\right) \mathbf{x}_{i} \cdot \nabla \mathbb{E}_{n}^{m}(\mathbf{r})\right) \\
& -12 \sum_{m=1}^{3}\left(\sum_{p=1}^{3} M_{p} f_{1}^{m, p}\right) I_{1}^{m}\left(a_{1}\right) \frac{h_{1} h_{2} h_{3}}{2 h_{m} a_{i}^{2}} x_{m} x_{i} \\
& -5 \sum_{m=1}^{5}\left(\sum_{p=1}^{3} M_{p} f_{2}^{m, p}\right) \frac{I_{2}^{m}\left(a_{1}\right)}{2 a_{i}^{2}}\left[3 x_{i} \mathbb{E}_{2}^{m}(\mathbf{r})+\mathbf{r} \cdot\left(x_{i} \nabla \mathbb{E}_{2}^{m}(\mathbf{r})\right)\right] \\
& \left.-\sum_{m=1}^{5} \sum_{p=1}^{3} C_{2}^{m, i} e_{2}^{m, p} \mathbf{x}_{p} \cdot\left(x_{i} \nabla \mathbb{E}_{2}^{m}(\mathbf{r})\right)\right]_{\rho=a_{1}}=0,
\end{aligned}
$$

where the $B_{n+}^{m p}$ and $B_{n-}^{m p}$ are known constants. In order to apply the orthogonal property of the harmonics, some terms have to be solely explained in terms of harmonics. They are $x_{p} \mathbf{x}_{i} \cdot \nabla \mathbb{E}_{n}^{m}(\mathbf{r}), \mathbf{x}_{i} \cdot \nabla \mathbb{E}_{n}^{m}(\mathbf{r}), x_{m} x_{i}, x_{i} \mathbb{E}_{n}^{m}(\mathbf{r})$ and $\mathbf{x}_{p} \cdot x_{i} \nabla \mathbb{E}_{n}^{m}(\mathbf{r})$. As mentioned before, details are not given here but can easily be found.

As a result, the following equation is obtained while applying orthogonality to harmonics of first order $E_{0}^{1}(\mu) E_{0}^{1}(\nu)$ :

$$
\begin{aligned}
\frac{4 \pi}{a_{1} a_{2} a_{3}} e_{0}^{1, i}-\frac{h_{1} h_{2} h_{3} a_{i}}{6 h_{i}} D_{1}^{i} \mathbf{f}_{1}^{i} \cdot \frac{\mathbf{M}}{4 \pi} \\
\quad-\left(\frac{\mathbb{F}_{2}^{2}\left(\mathbf{r}_{0}\right)}{\Lambda^{\prime}-a_{i}^{2}}-\frac{\mathbb{F}_{2}^{1}\left(\mathbf{r}_{0}\right)}{\Lambda-a_{i}^{2}}\right) \frac{M_{i}}{6\left(\Lambda-\Lambda^{\prime}\right)}-\sum_{q=1}^{3} \frac{\left(1-\delta_{q i}\right) h_{q} h_{i}}{\left(h_{1} h_{2} h_{3}\right)^{2}} \mathbb{F}_{2}^{i+q}\left(\mathbf{r}_{0}\right) M_{q}=0,
\end{aligned}
$$

where $i=1,2,3$.

For the second-order harmonics $E_{1}^{p}(\mu) E_{1}^{p}(\nu)$, with $p=1,2,3$, two different cases appear depending on whether $i=p$ or not. The three first equations provide the three constants

$$
e_{1}^{i, i}=\frac{h_{i} a_{i}^{2} a_{1} a_{2} a_{3}}{15 h_{1} h_{2} h_{3}}\left[\frac{\prod_{l=1}^{3}\left(\Lambda-a_{l}^{2}\right)}{\Lambda-a_{i}^{2}} D_{2}^{1} \mathbf{f}_{2}^{1}+\frac{\prod_{l=1}^{3}\left(\Lambda^{\prime}-a_{l}^{2}\right)}{\Lambda^{\prime}-a_{i}^{2}} D_{2}^{2} \mathbf{f}_{2}^{2}\right] \cdot \frac{\mathbf{M}}{(4 \pi)^{2}}
$$

and the following six equations describe the case $i \neq p$ with six unknown constants $e_{1}^{i, p}$ :

$$
h_{p} e_{1}^{i, p}+h_{i} e_{1}^{p, i}=\frac{1}{15} h_{1} h_{2} h_{3} a_{i} a_{p} a_{1} a_{2} a_{3} D_{2}^{i+p} \mathbf{f}_{2}^{i+p} \cdot \frac{\mathbf{M}}{(4 \pi)^{2}},
$$


where $i=1,2,3$ and $p=1,2,3$. Orthogonality for $E_{2}^{l}(\mu) E_{2}^{l}(\nu)$ harmonics, with $l=1,2$, provides

$$
\begin{aligned}
& \frac{(-1)^{l} h_{1} h_{2} h_{3}}{2 a_{1} a_{2} a_{3} h_{i}} \frac{\left[1-5 a_{1} a_{2} a_{3} I_{1}^{i}\left(a_{1}\right)\right]}{\left(\Lambda-\Lambda^{\prime}\right)\left(L-a_{i}^{2}\right)} \mathbf{M} \cdot \mathbf{f}_{1}^{i}+L\left(2 B_{2+}^{l, i}+D_{2}^{l} 4 \pi e_{2}^{l, i}\right) \\
& +\sum_{q=1}^{3} \frac{(-1)^{l}\left(1-\delta_{i q}\right)\left(h_{1} h_{2} h_{3}\right)^{2}}{3\left(\Lambda-\Lambda^{\prime}\right) h_{i} h_{q}}\left[\frac{a_{q}^{2}\left(2 B_{2-}^{i+q, q}+C_{2}^{i+q, q} e_{2}^{i+q, q}\right)}{\left(L-a_{q}^{2}\right)}-\frac{a_{i}^{2} C_{2}^{i+q, i} e_{2}^{i+q, q}}{\left(L-a_{i}^{2}\right)}\right] \\
& +\frac{(-1)^{l} a_{k}^{2}}{3\left(\Lambda-\Lambda^{\prime}\right)\left(L-a_{i}^{2}\right)}\left[\frac{4 B_{2-}^{1, i}}{\left(\Lambda-a_{i}^{2}\right)} \prod_{p=1}^{3}\left(\Lambda-a_{p}^{2}\right)+\frac{4 B_{2-}^{2, i}}{\left(\Lambda^{\prime}-a_{i}^{2}\right)} \prod_{p=1}^{3}\left(\Lambda^{\prime}-a_{p}^{2}\right)\right]=0, \\
& \text { where }(l, L)=(1, \Lambda) \text { or }\left(2, \Lambda^{\prime}\right) \text { and for } i=1, \cdots, 3,
\end{aligned}
$$

while orthogonality on $E_{2}^{6-i}(\mu) E_{2}^{6-i}(\nu)$ gives

$$
\sum_{q=1}^{3}\left(1-\delta_{i q}\right) h_{q}\left[2 B_{2-}^{6-q, q}+C_{2}^{6-q, q} e_{2}^{6-q, q}\right]+h_{i}\left[2 B_{2+}^{6-i, i}-\frac{a_{i}}{a_{1} a_{2} a_{3}} D_{2}^{6-i} e_{2}^{6-i, i}\right]=0,
$$

and the one with $E_{2}^{i+q}(\mu) E_{2}^{i+q}(\nu)$ results in

$$
\begin{gathered}
\frac{3 h_{i}\left[a_{i}^{2} a_{1} a_{2} a_{3}\left(a_{i}^{2}+4 a_{q}^{2}\right) I_{1}^{q}\left(a_{1}\right)\right]}{2 a_{i} a_{q} a_{1} a_{2} a_{3} h_{1} h_{2} h_{3}} \mathbf{M} \cdot \mathbf{f}_{1}^{q}+a_{i} a_{q}\left(2 B_{2+}^{i+q, i}+D_{2}^{i+q} 4 \pi e_{2}^{i+q, q}\right) \\
+\frac{h_{i} h_{q} a_{i} a_{q}}{\left(h_{1} h_{2} h_{3}\right)^{2}}\left[\frac{\left(h_{1} h_{2} h_{3}\right)^{2}}{h_{i} h_{q}} 2 B_{2-}^{i+q, i}-\left(h_{1} h_{2} h_{3}\right) h_{i} C_{2}^{6-i, i} e_{2}^{6-i, q}\right. \\
+\left(\frac{2 B_{2-}^{1, q}+C_{2}^{1, q} e_{2}^{1, q}}{\left(\Lambda-a_{i}^{2}\right)}-\frac{C_{2}^{1, i} e_{2}^{1, q}}{\left(\Lambda-a_{q}^{2}\right)}\right) 2 \prod_{l=1}^{3}\left(\Lambda-a_{l}^{2}\right) \\
\left.+\left(\frac{2 B_{2-}^{2, q}+C_{2}^{2, q} e_{2}^{2, q}}{\left(\Lambda^{\prime}-a_{i}^{2}\right)}-\frac{C_{2}^{2, i} e_{2}^{2, q}}{\left(\Lambda^{\prime}-a_{q}^{2}\right)}\right) 2 \prod_{l=1}^{3}\left(\Lambda^{\prime}-a_{l}^{2}\right)\right]=0,
\end{gathered}
$$

where $i=1,2,3$ and $q=1,2,3$, but $q \neq i$.

Equations coming from the first and the second boundary conditions have to both be satisfied and the unknown constants $e_{n}^{m, i}, d_{n}^{m}$ have to be found. Equation (79), using the expression of the $\mathbf{f}_{1}^{i}$ constant in (69), directly yields the three constants $e_{0}^{1, i}$ :

$$
e_{0}^{1, i}=\frac{h_{1} h_{2} h_{3}}{2 h_{i}} \frac{\mathbf{M} \cdot \mathbf{f}_{1}^{i}}{4 \pi} \quad(\text { for } i=1,2,3) .
$$

The $e_{1}^{i, i}$ solutions in (80) included in (74) and (76) provide the $d_{0}^{1}$ and $d_{2}^{l}$ (that are not involved elsewhere):

$$
\begin{gathered}
d_{0}^{1}=a_{1} a_{2} a_{3} \sum_{i=1}^{3} \frac{h_{1} h_{2} h_{3}}{h_{i}} I_{1}^{i}\left(a_{1}\right) e_{1}^{i, i} \\
d_{2}^{l}=\frac{-1}{D_{2}^{l}} \sum_{p=1}^{3}\left[A_{2}^{l, p}+(-1)^{l} \frac{h_{1} h_{2} h_{3}}{\left(\Lambda-\Lambda^{\prime}\right)} \frac{a_{p}^{2} C_{1}^{p, p}}{3 h_{p}\left(L-a_{p}^{2}\right)} e_{1}^{p, p}\right]
\end{gathered}
$$

with $(l, L)=(1, \Lambda)$ or $\left(2, \Lambda^{\prime}\right)$. 
Assuming that $e_{1}^{i, p}=e_{1}^{p, i}$ (in order to satisfy geometrical symmetry), equation (81) and equation (777) respectively from the second and first boundary conditions provide the $e_{1}^{i, p}$ and $d_{2}^{i+p}$ constants

$$
\begin{aligned}
& e_{1}^{i, p}=\frac{h_{1} h_{2} h_{3}}{3\left(h_{p}+h_{i}\right)}\left[a_{1} a_{2} a_{3}\left(a_{p}^{2}+a_{i}^{2}\right) I_{2}^{p+i}\left(a_{1}\right)-1\right] \frac{\mathbf{M} \cdot \mathbf{f}_{2}^{p+i}}{4 \pi}, \\
& d_{2}^{i+p}= \\
& -\frac{a_{i} a_{p}}{D_{2}^{p+i}\left(h_{1} h_{2} h_{3}\right)}\left[\left(h_{i} C_{1}^{p, i}+h_{p} C_{1}^{i, p}\right) e_{1}^{i, p}+\left(h_{i} B_{1}^{p, i}+h_{p} B_{1}^{i, p}\right)+\sum_{m=1}^{3} \frac{h_{1} h_{2} h_{3}}{a_{i} a_{p}} A_{2}^{p+i, m}\right],
\end{aligned}
$$

where $i=1,2,3$ and $p=1,2,3$, but $p \neq i$.

At this step, four equations have not been used (175), (82), (83) and (84)), and the constants $d_{1}^{i}$ and the vector constants $\mathbf{e}_{2}^{i}$ are still unknown. The vector constants $\mathbf{e}_{2}^{i}$ cannot be found in a simple analytical form using these equations; this is due to the truncation of the expansion at $n=2$. So one has decided to put the $\mathbf{e}_{2}^{i}$ vector constants to zero. It might be seen as a rather rude way to proceed but it can be justified by looking to the $\mathbf{H}_{2}$ expression in equation (32). If the expansion is stopped at $n=2$, the $d_{2}^{i}$ term is in $\nabla \mathbb{F}_{2}^{i}(\mathbf{r})$ and so has a behavior approximately similar to the harmonic $\mathbb{E}_{1}^{i}(\mathbf{r})$, while the $\mathbf{e}_{2}^{i}$ term is in $\mathbb{F}_{2}^{i}(\mathbf{r})$ (one order higher than the previous term). What is done here is a truncation at $n=2$ for the $d_{2}^{i}$ term but at $n=1$ for the $\mathbf{e}_{2}^{i}$ term in order to have the last term of the harmonic field possess a behavior similar to the $\mathbb{E}_{1}^{i}(\mathbf{r})$ harmonic.

Equation (75) provides the $d_{1}^{i}$ constants while $e_{2}^{i, p}=0$ for all $i=1, \cdots, 5$ and $p=$ $1,2,3$ :

$$
\begin{array}{r}
d_{1}^{i}=\frac{-1}{D_{1}^{i}}\left\{\sum_{p=1}^{3} A_{1}^{i, p}+\frac{a_{i} h_{i}}{h_{1} h_{2} h_{3}}\left[C_{0}^{1, i} e_{0}^{1, i}+B_{0}^{1, i}+\sum_{p=1}^{3} \frac{\left(1-\delta_{i p}\right)\left(h_{1} h_{2} h_{3}\right)^{2} a_{p}^{2} B_{2}^{i+p, p}}{5 h_{i} h_{p}}\right.\right. \\
\left.\left.\left.+\frac{2 \Lambda \prod_{l=1}^{3}\left(\Lambda-a_{l}^{2}\right) B_{2}^{1, i}}{5\left(\Lambda-a_{i}^{2}\right)}+\frac{2 \Lambda^{\prime} \prod_{l=1}^{3}\left(\Lambda^{\prime}-a_{l}^{2}\right) B_{2}^{2, i}}{5\left(\Lambda^{\prime}-a_{i}^{2}\right)}\right]\right\} \quad \text { (for } i=1,2,3\right) .
\end{array}
$$

\section{REFERENCES}

[1] J. Björkberg, G. Kristensson, Three-dimensional subterranean target identification by use of optimization techniques, PIER, vol. 15, 1997, pp. 141-164.

[2] B. Bourgeois, D. Legendre, M. Lambert, G. Hendrickson, Three Dimensional Electromagnetics, SEE, 1999, pp. 625-657.

[3] T. Yu, L. Carin, Analysis of the electromagnetic inductive response of a void in a conductingsoil background, IEEE Transactions on Geoscience and Remote Sensing, vol. 38, no. 3, 2000, pp. $1320-1327$.

[4] H. Huang, I. J. Won, Detecting metal objects in magnetic environments using a broadband electromagnetic method, Geophysics, vol. 68, no. 6, 2003, pp. 1877-1887. 
[5] X. Chen, K. O'Neill, B. E. Barrowes, T. M. Grzegorczyk, J. A. Kong, Application of a spheroidal mode approach and differential evolution in inversion of magneto-quasistatic data for UXO discrimination, Inverse Problems, vol. 20, no. 6, 2004, pp. 527-540.

[6] T. J. Cui, W. C. Chew, D. L. Wright, D. V. Smith, Three dimensional imaging of buried objects in very lossy earth by inversion of VETEM data, IEEE Transactions on Geoscience and Remote Sensing, vol. 41, no. 10, 2003, pp. 2197-2210.

[7] H. Tortel, Electromagnetic imaging of a three-dimensional perfectly conducting object using a boundary integral formulation, Inverse Problems, vol. 20, 2004, pp. 385-398. MR2065429 (2005c:78029)

[8] G. Perrusson, M. Lambert, D. Lesselier, A. Charalambopoulos, G. Dassios, Electromagnetic scattering by a triaxial homogeneous penetrable ellipsoid : low-frequency derivation and testing of the localized non-linear approximation, Radio Science, vol. 35, no. 2, 2000, pp. 463-481.

[9] G. L. Wang, W. C. Chew, T. J. Cui, D. L. Wright, D. V. Smith, 3D near-to-surface conductivity reconstruction by inversion of VETEM data using the distorted Born iterative method, Inverse Problems, vol. 20, 2004, pp. 195-216.

[10] C. O. Ao, H. Braunisch, K. O'Neill, J. A. Kong, Quasi-magnetostatic solution for a conducting and permeable spheroid with arbitrary excitation, IEEE Transactions on Geoscience and Remote Sensing, vol. 40, no. 4, 2002, pp. 887-897.

[11] G. Perrusson, P. Vafeas, D. Lesselier, Low-frequency modeling of the interaction of magnetic dipoles and ellipsoidal bodies in a conductive medium, 2004 URSI International Symposium on Electromagnetic Theory, Pisa, Proceedings, pp. 1017-1019 (+ CD-ROM), May, 2004.

[12] T. Habashy, R. Groom, B. Spies, Beyond the Born and the Rytov approximations: a non-linear approach to electromagnetic scattering, Journal of Geophysical Research, vol. 98, 1993, pp. 17591775 .

[13] E. W. Hobson, The Theory of Spherical and Ellipsoidal Harmonics, Chelsea, New York, 1965.

[14] G. Dassios, K. Kiriaki, The rigid ellipsoid in the presence of a low frequency elastic wave, Quarterly of Applied Mathematics, vol. XLIII, no. 4, 1986, pp. 435-456. MR846156 (87f:73021)

[15] P. Vafeas, G. Dassios, Stokes flow in ellipsoidal geometry, Journal of Mathematical Physics, vol. 47, 2006, 093102. MR2263654 (2007j:76051)

[16] P. Vafeas, G. Perrusson, D. Lesselier, Low-frequency solution for a perfectly conducting sphere in a conductive medium with dipolar excitation, PIER, vol. 49, 2004, pp. 87-111.

[17] P. Moon, D. E. Spencer, Field Theory Handbook, Springer-Verlag, Berlin, 1961. MR947546 (89i:00026) 